

\title{
Localized states in an unbounded neural field equation with smooth firing rate function: a multi-parameter analysis
}

Grégory Faye, James Rankin, Pascal Chossat

\section{- To cite this version:}

Grégory Faye, James Rankin, Pascal Chossat. Localized states in an unbounded neural field equation with smooth firing rate function: a multi-parameter analysis. [Research Report] RR-7872, INRIA. 2012, pp.31. hal-00665464

\section{HAL Id: hal-00665464 \\ https://hal.inria.fr/hal-00665464}

Submitted on 2 Feb 2012

HAL is a multi-disciplinary open access archive for the deposit and dissemination of scientific research documents, whether they are published or not. The documents may come from teaching and research institutions in France or abroad, or from public or private research centers.
L'archive ouverte pluridisciplinaire HAL, est destinée au dépôt et à la diffusion de documents scientifiques de niveau recherche, publiés ou non, émanant des établissements d'enseignement et de recherche français ou étrangers, des laboratoires publics ou privés. 
Localized states in an unbounded neural field equation with smooth firing rate function: a multi-parameter analysis

Grégory Faye, James Rankin, Pascal Chossat

RESEARCH

REPORT

$\mathbf{N}^{\circ} \mathbf{7 8 7 2}$

January 2012

Project-Team Neuromathcomp 



\title{
Inicia
}

\section{Localized states in an unbounded neural field equation with smooth firing rate function: a multi-parameter analysis}

\author{
Grégory Faye, James Rankin, Pascal Chossat \\ Project-Team Neuromathcomp
}

Research Report $\mathrm{n}^{\circ} 7872$ - January 2012 - 31 pages

\begin{abstract}
The existence of spatially localized solutions in neural networks is an important topic in neuroscience as these solutions are considered to characterize working (short-term) memory. We work with an unbounded neural network represented by the neural field equation with smooth firing rate function and a wizard hat spatial connectivity. Noting that stationary solutions of our neural field equation are equivalent to homoclinic orbits in a related fourth order ordinary differential equation, we apply normal form theory for a reversible Hopf bifurcation to prove the existence of localized solutions; further, we present results concerning their stability. Numerical continuation is used to compute branches of localized solution that exhibit snaking-type behaviour. We describe in terms of three parameters the exact regions for which localized solutions persist.
\end{abstract}

Key-words: Localized state, Neural field equation, Reversible Hopf-bifurcation, Normal form, Orbital stability, Numerical continuation

\section{RESEARCH CENTRE}

SOPHIA ANTIPOLIS - MÉDITERRANÉE

2004 route des Lucioles - BP 93

06902 Sophia Antipolis Cedex 


\section{Solutions localisées d'équations de champs neuronaux non bornés avec nonlinéarité de type sigmoidal}

Résumé : L'existence de solutions spatialement localisées dans les réseaux de neurones est un important sujet de recherche en neuroscience puisque ces solutions sont considérées comme étant une des caractéristiques de la mémoire à court terme. Dans cet article, nous travaillons avec un réseau de neurones non borné modélisé par une équation de champ neuronal avec une nonlinéarité de type sigmoidal ainsi qu'une connectivité spatiale de type "chapeau de sorcier". En remarquant que les solutions stationaires de notre équation de champ neuronal sont équivalentes à des orbites homoclines pour une équation différentielle d'ordre quatre associée, nous pouvons appliquer la théorie des formes normales pour une bifurcation de Hopf réversible et ainsi prouver l'existence de solutions localisées. Nous présentons également des résultats concernant leur stabilité. Nous utilisons des méthodes numériques pour continuer ces branches de solutions et présentons des diagrames de bifurcation qui "serpentent". Nous décrivons la région dans l'espace des paramètres pour laquelle les solutions localisées persistent.

Mots-clés : Solutions localisées, Equation de champs neuronaux, Bifurcation de Hopf réversible, Forme normale, Stabilité orbitale, Continuation numérique 


\section{Introduction}

In the past decades there has been a great deal of interest in the origin and properties of spatially localized structures in differential equations with applications in physics and neuroscience [1, 6 , $32,26,13,21,3$. The equation that is the most studied in pattern formation is the well-known generalized Swift-Hohenberg equation with either cubic/quintic or quadratic/cubic nonlinearities. For this particular example, the presence of localized steady states is a dynamical property: a bifurcation from the trivial state occurs for some value of a control parameter. These localized solutions are then viewed as homoclinic orbits to the trivial state.

In the neuroscience community, the canonical example is the Wilson-Cowan neural field equation [34, 1 .

$$
\tau \partial_{t} a(x, t)=-a(x, t)+\int_{-\infty}^{+\infty} w(x-y) S(a(y, t)) d y
$$

where $\tau$ is a temporal constant which we will take equal to $1 \mathrm{~ms}$ for mathematical simplicity. In this model, $a(x, t)$ represents the average membrane voltage of a neuronal population at spatial position $x$ and time $t$. The connectivity function $w(x)$ determines the coupling between elements and is assumed to be decaying with distance. $S$ is the firing rate function of the model. The study of localized solutions of equation (1.1) is an old and recurrent subject in the mathematical neuroscience community, where a number of different coupling functions $w$ and firing-rate functions $S$ have been used. The firing rate function can be an Heaviside step function [1, 32, 13, a piecewise linear function [21, 20, or a smooth function of sigmoidal type 26, 11, 17, 15. The connectivity function is always assumed to have a so-called "Mexican hat" or so-called "wizard hat" shape. Partial differential equation (PDE) methods [26, 25] can be employed to transform the neural field equation 1.1 into a partial differential equation involving high-order spatial derivatives.

Laing et al [26] numerically investigated the integro-differential equation (1.1) with

$$
S(x)=2 \exp \left(-r /(x-\theta)^{2}\right) H(x-\theta)
$$

where $H$ is the Heaviside step function and

$$
w(x)=e^{-b|x|}(b \sin |x|+\cos x) .
$$

The parameter $b$ governs the rate at which oscillations in $w$ decay with distance from $x=0$, the firing rate function $S$ has threshold $\theta$ and slope $r$. In [26, numerical simulations of (1.1) show the existence of spatially localized states. Numerical continuation techniques were used to follow, as the parameter $b$ is varied, branches of localized solutions of (1.1) and so-called "snaking" behaviour was found. This remarkable phenomenon, in which a series of fold bifurcations give rise to a hierarchy of localizaed solution branches with increasing number of bumps, has been studied extensively for the canonical Swift-Hohenberg equation [35, 4, 5, 29. Numerical investigations of [26] indicate that localized solutions of equation 1.1$]$ do not come into existence through a reversible-Hopf bifurcation from a constant solution as is the case for the Swift-Hohenberg equation. Although their system is reversible, the shape of the nonlinearity function $S$ in equation 1.2) ( $S$ is not analytical at $x=0$ ) renders impossible the application of tools from bifurcation theory. More recently, Elvin et al [15] used the Hamiltonian structure of equation (1.1) and developed numerical techniques to find all homoclinic orbits of the system.

The main motivation of this article is to complete the study of localized states of neural field equations on the unbounded real line initiated in [26, 15] by showing that these states are bifurcated branches of solutions emerging from the trivial state of equation (1.1) through reversible Hopf bifurcation with 1:1 resonance when the slope of the sigmoidal function is increased. To 
achieve this goal, we work with a wizard hat coupling function $w(x)$ (difference of exponential functions) and a smooth firing rate function which has non-vanishing derivatives at the fixed point of equation (1.1). As previously shown for the Swift-Hohenberg equation on the real line in [5, we calculate the normal form coefficients [23, 22, for the reversible 1:1 Hopf bifurcation and find a condition on the different parameters which ensures the existence of a pair of homoclinic branches. We present results on the stability of the bifurcating branches.

We use numerical continuation in order to extend the study with an investigation of snaking behaviour; these methods have been applied extensively for the Swift-Hohenberg equation [3, 4, 6, 28, 29, 35, and, in a few isolated cases for the neural field equation [26, 25, 11. Here, we use the continuation package AUTO [14] with the extension HOMCONT to follow homoclinic cycles corresponding to localized solutions under variation of system parameters. We confirm the solution structure determined analytically in this paper and reproduce previously observed snaking behaviour. Further, we identify the exact regions of parameter space for which localized solutions persist in terms of two parameters governing the shape of the nonlinearity $S$ and a third parameter governing the shaped of the connectivity function $w$.

This paper is organized as follows. In section 2 our model and notations are introduced. We explain in section 3 how stationary solutions of the neural field equation are equivalent to homoclinic orbits in a related fourth order ordinary differential equation and we show the existence of branches of localized solutions using normal form theory. Section 4 focuses on the stability of these branches of solutions. Finally, in section 5 we build on the theoretical results with a numerical investigation of localized solutions under the variation of three parameters.

\section{Wizard hat model}

We introduce a bifurcation paramter $\mu>0$ in the neural field equation 1.1)

$$
\begin{aligned}
\partial_{t} a(x, t) & =-a(x, t)+\int_{-\infty}^{+\infty} w(x-y) S(\mu a(y, t)) d y, \\
& =\mathcal{F}(a(x, t), \mu) .
\end{aligned}
$$

The firing rate function is either taken to be the sigmoidal function

$$
S(x)=\frac{1}{1+e^{-x+\theta}} \text { with } \theta>0
$$

or its shifted version via:

$$
S_{0}(x)=\frac{1}{1+e^{-x+\theta}}-\frac{1}{1+e^{\theta}} .
$$

We define a stationary solution to be time independent solution of (2.1), thus satisfies the equation:

$$
a(x)=\int_{-\infty}^{+\infty} w(x-y) S(\mu a(y)) d y .
$$

For a solution of (2.4), we define its region of excitation to be the set:

$$
R_{\mu}^{\theta}(a)=\{x \in \mathbb{R} \mid \mu a(x)>\theta\} .
$$

Following Amari's definition [1, a localized solution of (2.4) is a pattern $a(x)$ whose region of excitation consists of a finite disjoint union of bounded connected intervals and which decays to zero as $x$ goes to infinity. 


\subsection{Connectivity function}

In order to well define the convolutional part of the right hand side of equation (2.1), we have to take a connectivity function at least integrable over the real line. If $w$ belongs to $\mathrm{L}^{1}(\mathbb{R})$, the space of integrable functions on $\mathbb{R}$, we can define its Fourier transform $\widehat{w}$ as:

$$
\widehat{w}(\xi)=\int_{\mathbb{R}} w(x) e^{-i \xi x} d x
$$

If we further suppose that $\widehat{w} \in \mathrm{L}^{1}(\mathbb{R})$, then the inversion formula applies and we have:

$$
w(x)=\frac{1}{2 \pi} \int_{\mathbb{R}} \widehat{w}(\xi) e^{i \xi x} d \xi
$$

We now introduce some conditions on the connectivity function.

Hypothesis 1. We suppose that the following conditions are satisfied:

(i) $w, \widehat{w} \in L^{1}(\mathbb{R})$,

(ii) $w(0)>0$,

(iii) $\widehat{w}_{0} \stackrel{\text { def }}{=} \widehat{w}(0)<0$,

(iv) there exists $\xi_{c}>0$ such that $\widehat{w}_{c} \stackrel{\text { def }}{=} \widehat{w}\left( \pm \xi_{c}\right)=\max _{\xi \in \mathbb{R}} \widehat{w}(\xi)>0$,

(v) $\widehat{w}(\xi)=\frac{R\left(\xi^{2}\right)}{Q\left(\xi^{2}\right)}$ with $R, Q$ polinomial in $\xi^{2}$ satisfying $\operatorname{deg} R<\operatorname{deg} Q$.

The first condition tells us that the connectivity function and its Fourier transform are integrable over the real line such that we can apply the inversion formula for Fourier transform. The second condition ensures that the connectivity function is locally excitatory and the third condition that it is laterally inhibitory. The fourth condition says that $\widehat{w}(\xi)$ has two global maxima at $\pm \xi_{c}$. The condition $\widehat{w}_{c}>0$ is necessary for the stability analysis developed in 2.2 . Finally, the last condition ensures that the partial differential equation (PDE) method conducted in 3.1 can be applied.

In order to fix ideas, from now on, we will work with the following connectivity function (as used in [21, 20]):

$$
w(x)=b_{1} e^{-\sigma_{1}|x|}-b_{2} e^{-\sigma_{2}|x|},
$$

with Fourier transform given by

$$
\widehat{w}(\xi)=\int_{\mathbb{R}} w(x) e^{-i \xi x} d x=2\left(\frac{b_{1} \sigma_{1}}{\sigma_{1}^{2}+\xi^{2}}-\frac{b_{2} \sigma_{2}}{\sigma_{2}^{2}+\xi^{2}}\right) .
$$

The real constants $\left(b_{1}, b_{2}, \sigma_{1}, \sigma_{2}\right)$ are chosen such that all the conditions in Hypothesis 1 are satisfied.

Hypothesis 2. We assume that $\xi_{c}=1$.

We impose this condition in order to fix the period to $2 \pi$ of the critical modes which will bifurcate from the trivial state (see 2.2). Now, if we define

$$
\begin{aligned}
& \Gamma_{1}=2 \sigma_{1} \sigma_{2}\left(b_{1} \sigma_{2}-b_{2} \sigma_{1}\right), \\
& \Gamma_{2}=2\left(b_{1} \sigma_{1}-b_{2} \sigma_{2}\right),
\end{aligned}
$$

$\mathrm{RR} \mathrm{n}^{\circ} 7872$ 
then $\widehat{w}_{c}$ can be written:

$$
\widehat{w}_{c}=\frac{\Gamma_{1}+\Gamma_{2}}{1+\sigma_{1}^{2}+\sigma_{2}^{2}+\sigma_{1}^{2} \sigma_{2}^{2}} .
$$

The condition $\xi_{c}=1$, which is equivalent to $\left.\frac{d}{d \xi} \widehat{w}(\xi)\right|_{\xi=1}=0$, reduces to

$$
\Gamma_{1}\left(\sigma_{1}^{2}+\sigma_{2}^{2}+2\right)+\Gamma_{2}\left(1-\sigma_{1}^{2} \sigma_{2}^{2}\right)=0 .
$$

It is a straightforward computation to see that equations $(2.8)$ and 2.9$)$ imply that:

$$
\begin{gathered}
\sigma_{1}^{2} \sigma_{2}^{2}-\frac{\Gamma_{1}}{\widehat{w}_{c}}=1 \\
\sigma_{1}^{2}+\sigma_{2}^{2}-\frac{\Gamma_{2}}{\widehat{w}_{c}}=-2 .
\end{gathered}
$$

\subsection{Linear stability analysis of the trivial state}

The aim of this subsection is to show that equation (2.1) has always a unique trivial state that undergoes a bifurcation when increasing the slope $\mu$ of the sigmoidal function. We present the results for both shifted and unshifted sigmoidal function.

\subsubsection{Unshifted sigmoidal function $S$}

Equation (2.1) has the trivial solution $a_{0}(\mu)$ independent of time and space that satisfies:

$$
a_{0}(\mu)=\widehat{w}_{0} S\left(\mu a_{0}(\mu)\right) \text { for all } \mu>0 .
$$

The linearized equation around this trivial solution is:

$$
\partial_{t} a(x, t)=-a(x, t)+\mu S^{\prime}\left(\mu a_{0}(\mu)\right) \int_{\mathbb{R}} w(x-y) a(y, t) d y .
$$

Looking at perturbation of the form $a(x, t)=e^{\sigma t} e^{i \xi x}$, we obtain the following dispersion relation:

$$
\sigma(\xi)=-1+\mu S^{\prime}\left(\mu a_{0}(\mu)\right) \widehat{w}(\xi) .
$$

Lemma 2.1. There exists a unique solution $\left(\mu_{c}, a_{c}=a_{0}\left(\mu_{c}\right)\right)$ of:

$$
\left\{\begin{array}{l}
a_{c}=\widehat{w}_{0} S\left(\mu_{c} a_{c}\right) \\
1=\mu_{c} S^{\prime}\left(\mu_{c} a_{c}\right) \widehat{w}_{c} .
\end{array}\right.
$$

It is possible to express $\left(\mu_{c}, a_{c}\right)$ with the following analytic formulas:

$$
\begin{aligned}
\mu_{c} & =\frac{\widehat{w}_{0}^{2}}{a_{c} \widehat{w}_{c}\left(\widehat{w}_{0}-a_{c}\right)}, \\
a_{c} & =\frac{\widehat{w}_{0} \widehat{w}_{c} W\left(-\widehat{w}_{0} e^{-\frac{-\widehat{w}_{0}+\theta \widehat{w}_{c}}{\hat{w}_{c}}} / \widehat{w}_{c}\right)}{\widehat{w}_{c} W\left(-\widehat{w}_{0} e^{-\frac{-\widehat{w}_{0}+\theta \widehat{w}_{c}}{\widehat{w}_{c}}} / \widehat{w}_{c}\right)-\widehat{w}_{0}} .
\end{aligned}
$$

$W$ is the Lambert function which satisfies $W(x) e^{W(x)}=x$.

Proof. The proof is given in appendix $\mathrm{A}$.

From this Lemma, we deduce that for all $\mu<\mu_{c}$ the trivial solution $a_{0}(\mu)$ is stable. 


\subsubsection{Shifted sigmoidal function $S_{0}$}

In the case of the shifted sigmoidal function defined equation (2.3), the null solution $a_{0}=0$ independent of time and space is clearly a solution of $(2.4)$ for all $\mu>0$. Under the condition $\widehat{w}_{0}<0$ it is the unique stationary of time and space of (2.4). Following the same lines as for the unshifted sigmoidal function, for perturbation of the form $a(x, t)=e^{\sigma t} e^{i \xi x}$, we obtain for the dispersion relation:

$$
\sigma(\xi)=-1+\mu S_{0}^{\prime}(0) \widehat{w}(\xi)
$$

Then the critical value $\mu_{c}$ is given by:

$$
\mu_{c}=\frac{1}{S_{0}^{\prime}(0) \widehat{w}_{c}},
$$

and for all $\mu<\mu_{c}$ the null solution is stable.

\subsubsection{Choice of the sigmoidal function}

In order to simplify our notations, from now on, we work with the shifted sigmoidal function $S_{0}$ and denote for all $k \geq 1, S_{0}^{(k)}(0)=s_{k}$. Of course, all the results that we are going to present in the following sections are easily transportable to the unshifted case.

\subsection{Bifurcation of the trivial state for the full system}

The trivial state $a_{0}=0$ undergoes a bifurcation at the critical value $\mu=\mu_{c}$. Furthermore, as equation (2.1) is equivariant with respect to the translations and the symmetry $(x \rightarrow-x, a \rightarrow a)$, the bifurcation is a Pitchfork with $\mathbf{O}(2)$-symmetry [10, 22, We can apply the Lyapunov-Schmidt decomposition (see [10] for a review) on the Hilbert space $\mathcal{X}=\mathrm{L}_{p e r}^{2}[0,2 \pi]$, the set of $2 \pi$-periodic square integrable functions, in order to get a reduced equation on the two-dimensional space $\operatorname{Span}\left(e^{i x}, e^{-i x}\right)$. If we denote $\lambda=\mu-\mu_{c}$ and $v(x, t)=a(x, t)-a_{0}$, then the neural field equation (2.1) is transformed into:

$$
\partial_{t} v(x, t)=\mathbf{L}_{\mu_{c}} v(x, t)+\mathbf{R}(v(x, t), \lambda)
$$

where $\mathbf{L}_{\mu_{c}}$ and $\mathbf{R}$ are defined by

$$
\begin{aligned}
\mathbf{L}_{\mu_{c}} v(x, t) & =-v(x, t)+\mu_{c} s_{1} \int_{\mathbb{R}} w(x-y) v(y, t) d y \\
\mathbf{R}(v(x, t), \lambda) & =\int_{\mathbb{R}} w(x-y)\left[S_{0}\left(\left(\lambda+\mu_{c}\right) v(y, t)\right)-\mu_{c} s_{1} v(y, t)\right] d y
\end{aligned}
$$

and

$$
f_{0}(X, \lambda)=S_{0}\left(\left(\lambda+\mu_{c}\right) X\right)-\mu_{c} s_{1} X .
$$

It is straightforward to check that $\mathbf{R}(0,0)=D_{v} \mathbf{R}(0,0)=0$. We can write each solution of 2.17 on the form:

$$
v(x, t)=Z(t) e^{i x}+\overline{Z(t)} e^{i x}+\Phi(Z(t), \overline{Z(t)}, \lambda) .
$$

The reduced equation is then:

$$
\dot{Z}(t)=\left(\nu \lambda+\chi|Z(t)|^{2}\right) Z(t)+\text { h.o.t. }
$$

$\mathrm{RR} \mathrm{n}^{\circ} 7872$ 
Lemma 2.2. The coefficients of the reduced equation (2.19) are:

$$
\begin{aligned}
\nu & =\frac{1}{\mu_{c}} \\
\chi & =\frac{\mu_{c}^{3}}{s_{1}}\left[\frac{s_{3}}{2}+\frac{\mu_{c} s_{2}^{2}\left(19 \Gamma_{1}+4 \Gamma_{2}\right)}{18}\right] .
\end{aligned}
$$

Proof. The computation of the coefficients is postponed in appendix B.

It follows that close to the bifurcation point, for $\lambda \chi<0$, the amplitude $Z(t)$ is given by:

$$
Z_{\omega}(t)=\sqrt{-\frac{\lambda}{\mu_{c} \chi}} e^{i \omega}+\mathcal{O}\left(|\lambda|^{\frac{3}{2}}\right)
$$

for any phase $\omega$ on the circle $\mathbf{S}^{1}$. This phase can be identified to the translation iinvariance of equation 2.1). The bifurcation to this spatially periodic branch is subcritical $(\lambda<0)$ in $\chi>0$ and supercritical $(\lambda>0)$ in $\chi<0$.

\section{Reversible Hopf bifurcation with 1:1 resonance}

\subsection{PDE methods}

We assume that $v \rightarrow v(\cdot, t) \in \mathcal{C}^{1}\left(\mathbb{R}^{+}, \mathcal{H}^{4}(\mathbb{R})\right)$ is a solution of $(2.17)$, where $\mathcal{H}^{4}(\mathbb{R})$ is the Sobolev space defined as:

$$
\mathcal{H}^{4}(\mathbb{R})=\left\{u \in \mathrm{L}^{2}(\mathbb{R}) \mid \forall k \leq 4 \partial_{x^{k}}^{k} u \in \mathrm{L}^{2}(\mathbb{R})\right\} .
$$

Under this assumption, an application of Fourier transform of (2.17) gives:

$$
\left(\partial_{t}+1\right) \widehat{v}(\xi, t)=\widehat{w}(\xi)\left[\widehat{f_{0}(v, \lambda)}(\xi, t)+\mu_{c} s_{1} \widehat{v}(\xi, t)\right] .
$$

Using the inverse Fourier transform we obtain:

$$
\left(\partial_{t}+1\right) \mathcal{L}_{\mu_{c}}(v)=\mathcal{M}(v, \lambda)
$$

with $\mathcal{L}_{\mu_{c}}$ and $\mathcal{M}$ defined by

$$
\mathcal{L}_{\mu_{c}}(v)=\left(\sigma_{1}^{2} \sigma_{2}^{2}-\Gamma_{1} \mu_{c} s_{1}\right) v-\left(\sigma_{1}^{2}+\sigma_{2}^{2}-\Gamma_{2} \mu_{c} s_{1}\right) \partial_{x^{2}}^{2} v+\partial_{x^{4}}^{4} v
$$

and

$$
\mathcal{M}(v, \lambda)=\Gamma_{1} f_{0}(v, \lambda)-\Gamma_{2} \partial_{x^{2}}^{2}\left[f_{0}(v, \lambda)\right] .
$$

From equations 2.10 and the fact that $\mu_{c}=\left(s_{1} \widehat{w}_{c}\right)^{-1}$, the coefficients of $\mathcal{L}_{\mu_{c}}$ reduce to:

$$
\begin{aligned}
\sigma_{1}^{2} \sigma_{2}^{2}-\Gamma_{1} \mu_{c} s_{1} & =1, \\
\sigma_{1}^{2}+\sigma_{2}^{2}-\Gamma_{2} \mu_{c} s_{1} & =-2 .
\end{aligned}
$$

Note that equation (3.1) forms a fourth order reversible dynamical system in space: the equation is invariant under spatial reflection $(x \rightarrow-x, v \rightarrow v)$. We look for stationary solutions of equation (3.1) which staisfy

$$
\left\{\begin{aligned}
\mathcal{L}_{\mu_{c}}(v) & =\mathcal{M}(v, \lambda) \\
v & \in \mathcal{H}^{4}(\mathbb{R})
\end{aligned}\right.
$$


The spatial coordinate $x$ is recast as the time variable and the differential equation $(3.2)$ is now a four-dimensional system of first order ordinary differential equations (ODEs) which can be written:

$$
U^{\prime}=\mathcal{A} U+\mathcal{R}(U, \lambda)
$$

with $U=\left(u_{1}, u_{2}, u_{3}, u_{4}\right)^{\mathbf{T}}$ (note thet $u_{1}=v$ ) and

$$
\mathcal{A}=\left(\begin{array}{cccc}
0 & 1 & 0 & 0 \\
0 & 0 & 1 & 0 \\
0 & 0 & 0 & 1 \\
-1 & 0 & -2 & 0
\end{array}\right), \quad \mathcal{R}(U, \lambda)=\left(\begin{array}{c}
0 \\
0 \\
0 \\
\mathcal{R}_{4}\left(u_{1}, u_{2}, u_{3}, u_{4}, \lambda\right)
\end{array}\right)
$$

The fourth component of the nonlinear function $\mathcal{R}$ is given by

$$
\begin{aligned}
\mathcal{R}_{4}\left(u_{1}, u_{2}, u_{3}, u_{4}, \lambda\right) & =\Gamma_{1} f_{0}\left(u_{1}, \lambda\right)-\Gamma_{2}\left[\left(\lambda+\mu_{c}\right)^{2} u_{2}^{2} S_{0}^{\prime \prime}\left(\left(\lambda+\mu_{c}\right) u_{1}\right)-\mu_{c} s_{1} u_{3}\right. \\
& \left.+\left(\lambda+\mu_{c}\right) u_{3} S_{0}^{\prime}\left(\left(\lambda+\mu_{c}\right) u_{1}\right)\right] .
\end{aligned}
$$

Furthermore, we have the following Taylor expansion of $\mathcal{R}$ at $\left(U=0_{\mathbb{R}^{4}}, \lambda=0\right)$

$$
\begin{aligned}
\mathcal{R}_{1,1}(U, \lambda) & =\lambda s_{1}\left(0,0,0, \Gamma_{1} u_{1}-\Gamma_{2} u_{3}\right)^{\mathbf{T}} \\
\mathcal{R}_{2,0}(U, U) & =\frac{\mu_{c}^{2} s_{2}}{2}\left(0,0,0, \Gamma_{1} u_{1}^{2}-\Gamma_{2}\left(2 u_{2}^{2}+2 u_{1} u_{3}\right)\right)^{\mathbf{T}} \\
\mathcal{R}_{3,0}(U, U, U) & =\frac{\mu_{c}^{3} s_{3}}{6}\left(0,0,0, \Gamma_{1} u_{1}^{3}-\Gamma_{2}\left(6 u_{1} u_{2}^{2}+3 u_{1}^{2} u_{3}\right)\right)^{\mathbf{T}} .
\end{aligned}
$$

\subsection{Reversible-Hopf bifurcation}

The associated linear problem of equation $(3.3)$ is

$$
U^{\prime}=\mathcal{A} U+\mathcal{R}_{1,1}(U, \lambda) .
$$

Eigenvalues of the linear problem statisfy the characteristic equation:

$$
X^{4}+\left(2+s_{1} \lambda \Gamma_{2}\right) X^{2}+1-s_{1} \lambda \Gamma_{1}=0 .
$$

To the leading order in $\lambda$ the discriminant of equation (3.5) seen as a quadratic equation in $X^{2}$ is

$$
\Delta(\lambda)=4 s_{1}\left(\Gamma_{1}+\Gamma_{2}\right) \lambda+o(\lambda)
$$

From equation $(2.8)$, we have $\operatorname{sign}\left(\Gamma_{1}+\Gamma_{2}\right)=\operatorname{sign}\left(\widehat{w}_{c}\right)>0$. Then, for $\lambda<0$ there exists four complex eigenvalues with real part symmetric with respect to the imaginary axis, such that the trivial state is hyperbolic with two stables eigenvalues and two unstable eigenvalues. In contrast, for $\lambda>0$ all the eigenvalues lie on the imaginary axis and the trivial state is no longer hyperbolic. At $\lambda=0$, there is a pair of imaginary eigenvalues $\pm i$ of double multiplicity. The bifurcation at $\lambda=0$ is thus a Hopf bifurcation in a reversible system with 1:1 (spatial) resonance. 


\subsection{Normal form theory}

In the following, we adopt the formalism of [23, 22] to study the reversible Hopf bifurcation. We start by constructing a suitable basis of $\mathbb{R}^{4}$ and we denote $\mathbf{S}$ the symmetry:

$$
\mathbf{S}=\left(\begin{array}{cccc}
1 & 0 & 0 & 0 \\
0 & -1 & 0 & 0 \\
0 & 0 & 1 & 0 \\
0 & 0 & 0 & -1
\end{array}\right) \text { with } \mathbf{S}^{2}=\mathbb{I}_{\mathbb{R}^{4}}
$$

Let $\zeta_{0}=(1, i,-1,-i)^{\mathbf{T}}$ be an eigenvector of $\mathcal{A}$ which satisfies:

$$
\left(\mathcal{A}-i \mathbb{I}_{\mathbb{R}^{4}}\right) \zeta_{0}=0 \text { and } \mathbf{S} \zeta_{0}=\bar{\zeta}_{0}
$$

and let $\zeta_{1}=(0,1,2 i,-3)^{\mathbf{T}}$ be a generalized eigenvector that is:

$$
\left(\mathcal{A}-i \mathbb{I}_{\mathbb{R}^{4}}\right) \zeta_{1}=\zeta_{0} \text { and } \mathbf{S} \zeta_{1}=-\bar{\zeta}_{1} .
$$

Then $\left(\operatorname{Re} \zeta_{0}, \operatorname{Im} \zeta_{0}, \operatorname{Re} \zeta_{1}, \operatorname{Im} \zeta_{1}\right)$ is a basis of $\mathbb{R}^{4}$ with $\zeta_{0}, \zeta_{1}$ generalized eigenvectors of $\mathcal{A}$. In this basis, we represent a vector $U \in \mathbb{R}^{4}$ by $(A, B, \bar{A}, \bar{B})$,

$$
U=A \zeta_{0}+B \zeta_{1}+\overline{A \zeta_{0}}+\overline{B \zeta_{1}}
$$

with $A, B \in \mathbb{C}$.

Lemma 3.1 (Normal form). If we rewrite equation (3.3) as

$$
U^{\prime}=\mathcal{A} U+\mathcal{R}(U, \lambda)=\mathbf{F}(U, \lambda)
$$

then the vector field $\mathbf{F}$ is of class $\mathbb{C}^{k}, k \geq 3$, in a neighborhood of $(0,0) \in \mathbb{R}^{4} \times \mathbb{R}$ satisfying $\mathbf{F}(0,0)=0$ and such that $\mathbf{S}$ anticommutes with $\mathbf{F}$. For any integer $p, 2 \leq p \leq k$, there exist neighborhoods $\mathcal{V}_{1}$ and $\mathcal{V}_{2}$ of 0 in $\mathbb{R}^{4}$ and $\mathbb{R}$, respectively, and for any $\lambda \in \mathcal{V}_{2}$ there is a polynomial map $\Psi(\cdot, \lambda): \mathbb{R}^{4} \rightarrow \mathbb{R}^{4}$ of degree $p$ with the following properties:

1. The coefficients of the monomials of degree $q$ in $\Psi(\cdot, \lambda)$ are functions of $\lambda$ of class $\mathbb{C}^{k-q}$,

$$
\Psi(0,0,0,0,0)=0, \quad \partial_{(A, B, \bar{A}, \bar{B})} \Psi(0,0,0,0,0)=0,
$$

and

$$
\mathbf{S} \Psi(A, B, \bar{A}, \bar{B}, \lambda)=\Psi(\bar{A},-\bar{B}, A,-B, \lambda) .
$$

2. For $(A, B, \bar{A}, \bar{B}) \in \mathcal{V}_{1}$, the changes of variables

$$
U=A \zeta_{0}+B \zeta_{1}+\overline{A \zeta_{0}}+\overline{B \zeta_{1}}+\Psi(A, B, \bar{A}, \bar{B}, \lambda)
$$

transforms the equation (3.3) into the normal form:

$$
\begin{aligned}
\frac{d A}{d t}= & i A+B+i A P\left(|A|^{2}, \frac{i}{2}(A \bar{B}-\bar{A} B), \lambda\right)+\rho_{A}(A, B, \bar{A}, \bar{B}, \lambda) \\
\frac{d B}{d t}= & i B+i B P\left(|A|^{2}, \frac{i}{2}(A \bar{B}-\bar{A} B), \lambda\right)+A Q\left(|A|^{2}, \frac{i}{2}(A \bar{B}-\bar{A} B), \lambda\right) \\
& +\rho_{B}(A, B, \bar{A}, \bar{B}, \lambda)
\end{aligned}
$$


where $P$ and $Q$ are real-valued polynomials of degree $p-1$ in $(A, B, \bar{A}, \bar{B})$. The remainders $\rho_{A}$ and $\rho_{B}$ are of class $\mathbb{C}^{k}$, and satisfy

$$
\begin{aligned}
& \rho_{A}(\bar{A},-\bar{B}, A,-B, \lambda)=-\rho_{A}(A, B, \bar{A}, \bar{B}, \lambda), \\
& \rho_{B}(\bar{A},-\bar{B}, A,-B, \lambda)=\rho_{B}(A, B, \bar{A}, \bar{B}, \lambda)
\end{aligned}
$$

with the estimate

$$
\left|\rho_{A}(A, B, \bar{A}, \bar{B}, \lambda)\right|+\left|\rho_{B}(A, B, \bar{A}, \bar{B}, \lambda)\right|=o\left((|A|+|B|)^{p}\right) .
$$

Proof. See Haragus-Iooss [22].

Moreover, the expansions of $P$ and $Q$ in the normal form are given by:

$$
\begin{gathered}
P\left(|A|^{2}, \frac{i}{2}(A \bar{B}-\bar{A} B), \lambda\right)=\alpha \lambda+\beta|A|^{2}+\frac{i \gamma}{2}(A \bar{B}-\bar{A} B)+0\left(\left(|\lambda|+(|A|+|B|)^{2}\right)^{2}\right) \\
Q\left(|A|^{2}, \frac{i}{2}(A \bar{B}-\bar{A} B), \lambda\right)=c_{1}^{1} \lambda+c_{3}^{0}|A|^{2}+\frac{i c}{2}(A \bar{B}-\bar{A} B)+0\left(\left(|\lambda|+(|A|+|B|)^{2}\right)^{2}\right) .
\end{gathered}
$$

We wish to determine the different coefficients that appear in the expansions of $P$ and $Q$. The expression of each coefficient is given in the following lemma.

Lemma 3.2. The coefficients $\alpha, \beta, \gamma, c_{1}^{1}, c_{3}^{0}$ and $c$ in the expansions of $P$ and $Q$ in equations (3.7) and 3.8 are

$$
\begin{aligned}
\alpha & =\frac{s_{1}\left(\Gamma_{2}-\Gamma_{1}\right)}{8}, \\
\beta & =\frac{\mu_{c}^{3}}{32}\left[3 s_{3}\left(\Gamma_{2}-\Gamma_{1}\right)-\frac{\mu_{c} s_{2}^{2}\left(4 \Gamma_{2}^{2}+187 \Gamma_{1}^{2}+29 \Gamma_{1} \Gamma_{2}\right)}{27}\right], \\
\gamma & =-\frac{\mu_{c}^{4} s_{2}^{2}\left(36 \Gamma_{1}^{2}+4 \Gamma_{1} \Gamma_{2}+7 \Gamma_{2}^{2}\right)}{162}, \\
c_{1}^{1} & =-\frac{s_{1}\left(\Gamma_{1}+\Gamma_{2}\right)}{4}, \\
c_{3}^{0} & =-\frac{\mu_{c}^{3}\left(\Gamma_{1}+\Gamma_{2}\right)}{4}\left[\frac{s_{3}}{2}+\frac{\mu_{c} s_{2}^{2}\left(19 \Gamma_{1}+4 \Gamma_{2}\right)}{18}\right], \\
c & =\frac{\mu_{c}^{3}}{16}\left[s_{3}\left(\Gamma_{2}-\Gamma_{1}\right)-\frac{\mu_{c} s_{2}^{2}\left(41 \Gamma_{1}^{2}-209 \Gamma_{1} \Gamma_{2}-52 \Gamma_{2}^{2}\right)}{27}\right] .
\end{aligned}
$$

The proof of lemma 3.2 is let in appendix C. We can note that, as expected, $\chi=\frac{c_{3}^{0}}{\mu_{c} c_{1}^{1}}$. Moreover, the coefficients are in agreement with those computed for the Swift-Hohenberg equation with quadratic/cubic nonlinearity [3, 4, 5].

\subsection{Existence of homoclinic orbits}

We are now able to state the main result of this part.

Theorem 3.3 (Existence). If $c_{3}^{0}<0$ and $\lambda<0$, in a neighborhood of the symmetric equilirbrium $a_{0}$ and for sufficiently small $\lambda$, there is a pair of reversible homoclinic orbits to $a_{0}$. 
Proof. The proof is a direct application of Theorem 3.21 in 22 with $c_{1}^{1}<0$ in Lemma 3.1

Under the conditions stated in the theorem, the homoclinic orbits of the normal form (3.6) truncated at cubic order are given in polar coordinates $A=r_{0} e^{i\left(t+\varphi_{0}\right)}, B=r_{1} e^{i\left(t+\varphi_{1}\right)}$ by

$$
\begin{aligned}
& r_{0}(t)=\sqrt{\frac{-2 c_{1}^{1} \lambda}{c_{3}^{0}}} \operatorname{sech}\left(t \sqrt{c_{1}^{1} \lambda}\right), \quad r_{1}=\left|r_{0}^{\prime}\right| \\
& \varphi_{1}-\varphi_{0} \in\{0, \pi\}, \quad \varphi_{0}(t)=\alpha \lambda t-\frac{2 \beta \sqrt{c_{1}^{1} \lambda}}{c_{3}^{0}} \tanh \left(t \sqrt{c_{1}^{1} \lambda}\right)+\phi .
\end{aligned}
$$

For sufficiently small $\lambda, \lambda<0$, the localized solution of 2.1 can be approximated by

$$
a(x)=2 \sqrt{\frac{-2 c_{1}^{1} \lambda}{c_{3}^{0}}} \operatorname{sech}\left(t \sqrt{c_{1}^{1} \lambda}\right) \cos (x+\phi)+\mathcal{O}(\lambda) .
$$

This family of localized solutions is parametrized by $\phi \in \mathbf{S}^{1}$, which controls the phase of the pattern within the $\operatorname{sech}(\cdot)$ envelope. Within the asymptotics this phase remains arbitrary, however it is known that this is no longer the case once terms beyond all orders are included [30, 24, 7]. These terms break the rotational invariance of the envelope equation and result in a weak flow on the circle $\mathbf{S}^{1}$. This flow in turn selects specific values of the phase $\phi=0$ and $\phi=\pi$. Indeed these phases are the only that preserve the revesbility symmetry $(x \rightarrow-x, v \rightarrow v)$. It follows that the two branches of homoclinic orbits given in Theorem 3.3 or equivalently the two branches of localized states bifurcate subcritically from $\lambda=0\left(c_{1}^{1}<0\right.$ in Lemma 3.1). This is illustrated in Figure 2. Along the $\phi=0$ branch, also called up branch, the midpoint $(x=0)$ of the localized state is always a local maximum, while along the $\phi=\pi$ branch, also called down branch, the midpoint is always a local minimum.

Finally, as $\chi=\frac{c_{3}^{0}}{\mu_{c} c_{1}^{1}}$ and $c_{1}^{1}<0$, we deduce that the condition $c_{3}^{0}<0$ is equivalent to $\chi>0$. From the discussion in 2.3 , there is also a subcritical bifurcation from the trivial state of a branch of spatially periodic solutions at $\lambda=0$ of equation $(2.1)$.

\section{$4 \quad$ Stability of localized solutions}

\subsection{Asymptotic stability}

In this section we denote $U_{0}^{\mu} \in \mathcal{H}^{4}(\mathbb{R})$ a localized solution of equation $(2.4)$ for a fixed value $\mu$ of the slope of the sigmoidal function. We linearize equation (2.1) around this localized solution:

$$
\partial_{t} a(x, t)=-a(x, t)+\mu \int_{\mathbb{R}} w(x-y) S_{0}^{\prime}\left(\mu U_{0}^{\mu}(y)\right) a(y, t) d y .
$$

We look for perturbation of the form $a(x, t)=p(x) e^{\sigma t}$, with $p \in \mathcal{H}^{4}(\mathbb{R})$, and obtain

$$
(\sigma+1) p(x)=\mu \int_{\mathbb{R}} w(x-y) S_{0}^{\prime}\left(\mu U_{0}^{\mu}(y)\right) p(y) d y
$$

Due to the translation invariance of equation (2.1) $\left(\sigma=0, \partial_{x} U_{0}^{\mu}\right)$ is always solution of the above equation. It follows that $U_{0}^{\mu}$ cannot be asymptotically stable. Nevertheless, it is possible to define a notion of stability adapted to this problem as we will now show. Let $\mathcal{T}_{\rho}$ be the transformation on $u \in \mathcal{H}^{4}(\mathbb{R})$ such that $\mathcal{T}_{\rho} u(x)=u(x+\rho)(\rho \in \mathbb{R})$. Then $\mathcal{T}_{\rho}$ commutes with equation (2.1) for 
all $\rho \in \mathbb{R}$. We define the " $\mathcal{T}$-orbit" of the stationary localized solution $U_{0}^{\mu} \in \mathcal{H}^{4}(\mathbb{R})$ of equation (2.4) by

For all $u, v \in \mathrm{L}^{2}(\mathbb{R})$, we set:

$$
\mathcal{O}=\left\{\mathcal{I}_{\rho} U_{0}^{\mu} \mid \rho \in \mathbb{R}\right\}
$$

$$
\langle u, v\rangle=\int_{\mathbb{R}} u(x) v(x) d x .
$$

We can now define the normal slice $\mathcal{N}$ to $\mathcal{O}$ at $U_{0}^{\mu}$ as the set:

$$
\mathcal{N}=\left\{v \in \mathrm{L}^{2}(\mathbb{R}) \mid\left\langle\partial_{x} U_{0}^{\mu}, v\right\rangle=0\right\} \subset \mathrm{L}^{2}(\mathbb{R}) .
$$

Remark 4.1. Note that $U_{0}^{\mu} \in \mathcal{N}$. Indeed:

$$
\left\langle\partial_{x} U_{0}^{\mu}, U_{0}^{\mu}\right\rangle=\int_{\mathbb{R}} \partial_{x} U_{0}^{\mu}(x) U_{0}^{\mu}(x) d x=\frac{1}{2}\left[\left(U_{0}^{\mu}(x)\right)^{2}\right]_{-\infty}^{+\infty}=0,
$$

because $U_{0}^{\mu} \in L^{2}(\mathbb{R})$.

Then we have the following decomposition.

Lemma 4.1. Let $\mathcal{V}$ a neighbourhood of $U_{0}^{\mu}$ in $L^{2}(\mathbb{R})$, then any $V \in \mathcal{V}$ can be decomposed into

$$
V=\mathcal{T}_{\rho}\left(U_{0}^{\mu}+Y\right)
$$

where $Y \in \mathcal{N}$ and $\rho \in \mathbb{R}$.

Proof. For $V \in \mathcal{V}$, we define the function $f$ as

$$
f: \rho \rightarrow f(\rho)=\left\langle\mathcal{T}_{-\rho} U_{0}^{\mu}, V\right\rangle=\int_{\mathbb{R}} U_{0}^{\mu}(x-\rho) V(x) d x .
$$

(i) We know that $\mathcal{C}_{c}^{\infty}(\mathbb{R})$, the set of differentiable functions of compact support, is dense in $\mathrm{L}^{2}(\mathbb{R})\left[2\right.$. Then, there exists a sequence $V_{n} \in \mathcal{C}_{c}^{\infty}(\mathbb{R})$, such that $V_{n} \underset{n \rightarrow+\infty}{\longrightarrow} V$ in $\mathrm{L}^{2}(\mathbb{R})$. We define $f_{n}$ as

For all $\rho \in \mathbb{R}$, we have

$$
f_{n}: \rho \rightarrow f_{n}(\rho)=\left\langle\mathcal{T}_{-\rho} U_{0}^{\mu}, V_{n}\right\rangle
$$

$$
\left|f_{n}(\rho)-f(\rho)\right| \leq\left\|U_{0}^{\mu}\right\|_{\mathrm{L}^{2}(\mathbb{R})}\left\|V_{n}-V\right\|_{\mathrm{L}^{2}(\mathbb{R})} \underset{n \rightarrow+\infty}{\longrightarrow} 0,
$$

where $\|\cdot\|_{L^{2}(\mathbb{R})}$ is the norm associated to the scalar product 4.8). This implies that $f_{n}$ uniformaly converges to $f$. Because $V_{n} \in \mathcal{C}_{c}^{\infty}(\mathbb{R})$, we deduce that $f_{n}(\rho) \underset{\rho \rightarrow \pm \infty}{\longrightarrow} 0$ and then $f(\rho) \underset{\rho \rightarrow \pm \infty}{\longrightarrow} 0$.

(ii) Moreover, from the Sobolev inequality [2, the injection $\mathcal{H}^{m}(\mathbb{R}) \subset \mathcal{C}^{m-1}(\mathbb{R})$ with $m \geq 1$, is continuous. Then $U_{0}^{\mu} \in \mathcal{H}^{4}(\mathbb{R}) \subset \mathcal{C}^{3}(\mathbb{R}) \subset \mathrm{L}_{l o c}^{1}(\mathbb{R})$ where $\mathrm{L}_{l o c}^{1}(\mathbb{R})$ is the space of functions which are integrable on any compact subset of $\mathbb{R}$. As $U_{0}^{\mu}(x)=U_{0}^{\mu}(-x)$, it is straightforward that $f_{n}(\rho)=V_{n} * U_{0}^{\mu}(\rho)$, where $*$ is the convolution on the real line. From the property of the convolution, $f_{n}$ is $\mathcal{C}^{\infty}$ on $\mathbb{R}$ for all $n \geq 1$ and it is easy to see that:

$$
f_{n}^{\prime}(\rho)=-\left\langle\mathcal{T}_{-\rho} \partial_{x} U_{0}^{\mu}, V_{n}\right\rangle .
$$

For all $\rho \in \mathbb{R}$, we have

$$
\left|f_{n}^{\prime}(\rho)+\left\langle\mathcal{T}_{-\rho} \partial_{x} U_{0}^{\mu}, V\right\rangle\right| \leq\left\|\partial_{x} U_{0}^{\mu}\right\|_{\mathrm{L}^{2}(\mathbb{R})}\left\|V_{n}-V\right\|_{\mathrm{L}^{2}(\mathbb{R})} \underset{n \rightarrow+\infty}{\longrightarrow} 0 .
$$

This implies that $f_{n}^{\prime}$ uniformly converges to the function $\rho \rightarrow-\left\langle\mathcal{T}_{-} \partial_{x} U_{0}^{\mu}, V\right\rangle$. As a consequence $f$ is $\mathcal{C}^{1}$ and $f^{\prime}(\rho)=-\left\langle\mathcal{T}_{-\rho} \partial_{x} U_{0}^{\mu}, V\right\rangle$. 
(iii) We can now complete the proof of the lemma by introducing the function $g$ :

$$
g(\rho)=\left\langle\mathcal{T}_{-\rho} U_{0}^{\mu}-V, \mathcal{T}_{-\rho} U_{0}^{\mu}-V\right\rangle=\left\|U_{0}^{\mu}\right\|_{\mathrm{L}^{2}(\mathbb{R})}^{2}+\|V\|_{\mathrm{L}^{2}(\mathbb{R})}^{2}-2 f(\rho) .
$$

We know that $g$ is $\mathcal{C}^{1}(\mathbb{R})$ and $g(\rho) \underset{\rho \rightarrow \pm \infty}{\longrightarrow}\left\|U_{0}^{\mu}\right\|_{\mathrm{L}^{2}(\mathbb{R})}^{2}+\|V\|_{\mathrm{L}^{2}(\mathbb{R})}^{2}$. This implies that $g$ has a minimum at $\rho=\rho_{0} \in \mathbb{R}$ where $g^{\prime}\left(\rho_{0}\right)=0$. This is equivalent to

$$
0=f^{\prime}\left(\rho_{0}\right)=-\left\langle\mathcal{T}_{-\rho_{0}} \partial_{x} U_{0}^{\mu}, V\right\rangle=-\left\langle\partial_{x} U_{0}^{\mu}, \mathcal{T}_{\rho_{0}} V\right\rangle
$$

We deduce that $\mathcal{T}_{\rho_{0}} V-U_{0}^{\mu} \in \mathcal{N}$, this proves the existence of $Y \in \mathcal{N}$ such that $\mathcal{T}_{\rho_{0}} V=$ $U_{0}^{\mu}+Y$.

We can apply the previous Lemma and decompose any solution $V \in \mathcal{V}$ of equation (2.1) as

$$
V(x, t)=\mathcal{T}_{\rho(t)}\left(U_{0}^{\mu}(x)+Y(x, t)\right)
$$

where $t \rightarrow \rho(t) \in \mathcal{C}^{1}(\mathbb{R})$ and $t \rightarrow Y(x, t) \in \mathcal{C}^{1}(\mathbb{R}, \mathcal{N})$. Replacing $V(x, t)$ into equation (2.1) and thanks to the translational equivariance of $\mathcal{F}$, we obtain the new equation

$$
\partial_{t} Y(x, t)+\rho^{\prime}(t) \partial_{x} U_{0}^{\mu}(x)+\rho^{\prime}(t) \partial_{x} Y(x, t)=\mathcal{F}\left(U_{0}^{\mu}(x)+Y(x, t), \mu\right) .
$$

We shall now decompose equation 4.8 into two parts: one part on the tangent space $T_{U_{0}^{\mu}} \mathcal{O}=$ $\operatorname{Span}\left(\partial_{x} U_{0}^{\mu}\right)$, which will solve $\rho^{\prime}(t)$, and the remaining part in the normal slice $\mathcal{N}$ to $\mathcal{O}$ at $U_{0}^{\mu}$, which will contain the relevant information about the dynamics near $\mathcal{O}$. We define a projection $\mathbf{P}$ in $\mathcal{H}^{4}(\mathbb{R})$ onto $T_{U_{0}^{\mu}} \mathcal{O}$ by

$$
\mathbf{P} u=\left\langle u, \partial_{x} U_{0}^{\mu}\right\rangle \frac{\partial_{x} U_{0}^{\mu}}{\left\|\partial_{x} U_{0}^{\mu}\right\|}
$$

Let us now apply $\mathbf{P}$ to equation (4.8). Then, since $Y \perp T_{U_{0}^{\mu}} \mathcal{O}$, we obtain the following equation

$$
\rho^{\prime}(t)\left(1+\mathbf{P} \partial_{x} Y(x, t)\right)=\mathbf{P} \mathcal{F}\left(U_{0}^{\mu}(x)+Y(x, t), \mu\right) .
$$

Since $U_{0}^{\mu}+Y$ is taken in a neighbourhood of $U_{0}^{\mu}, Y$ is small in norm, such that the left hand side of equation 4.10 is invertible. Therefore $\rho^{\prime}(t)$ can be solved in function of $Y$ and $\mu$ :

$$
\rho^{\prime}(t)=\left(1+\mathbf{P} \partial_{x} Y(x, t)\right)^{-1} \mathbf{P} \mathcal{F}\left(U_{0}^{\mu}(x)+Y(x, t), \mu\right) \text { for } Y \in \mathcal{V} \cap \mathcal{N} .
$$

It follows that $\rho$ is completely determined as a function of $Y$ and $\mu$.

The remaining equation in the normal slice $\mathcal{N}$ reads as

$$
\begin{aligned}
\partial_{t} Y(x, t) & =(I d-\mathbf{P})\left[\mathcal{F}\left(U_{0}^{\mu}(x)+Y(x, t), \mu\right)-\rho^{\prime}(t) \partial_{x} Y(x, t)\right], \\
& =\mathcal{G}(Y(x, t), \mu) .
\end{aligned}
$$

This decomposition is illustrated in Figure 1. It follows that the dynamics near the relative equilibrium $U_{0}^{\mu}$ is completely determined by the asymptotic behavior of the solutions of (4.11). If in particular $Y=0$ is asymptotically stable for (4.11), then it follows from the Lemma 4.1 that the $\mathcal{T}_{\rho}$-orbit $\mathcal{O}$ is an attractor for equation 2.1 . This justifies the following definition.

Definition 4.2. A localized solution $U_{0}^{\mu} \in \mathcal{H}^{4}(\mathbb{R})$ of equation $(2.1)$ is orbitally stable, if for any initial condition of the form $V_{0}=\mathcal{T}_{\rho_{0}}\left(U_{0}^{\mu}+Y_{0}\right), \rho_{0} \in \mathbb{R}$ and $Y_{0}$ close to $\mathcal{O}$ in $\mathcal{N}$, the solution $V(t)$ of (2.1) such that $V(0)=V_{0}$ satisfies $V(t)=\mathcal{T}_{\rho(t)}\left(U_{0}^{\mu}+Y(t)\right)$ with $Y(t)$ solution of equation 4.11) and $Y(t) \underset{t \rightarrow+\infty}{\longrightarrow} 0$ in $\mathcal{N}$. 


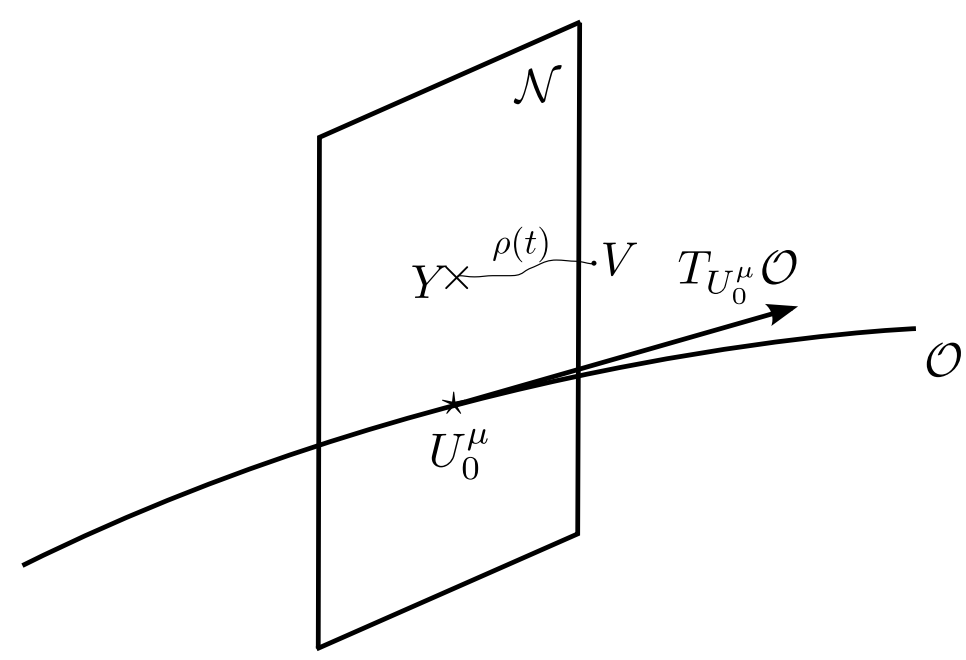

Figure 1: Decomposition of the flow near the relative equilibrium $U_{0}^{\mu}$.

If the spectrum of the linearized operator $\left.D_{Y} \mathcal{G}(Y, \mu)\right|_{Y=0}$ lies entirely in the half plane $\{\Re(z) \leq$ $\xi<0\}$, the localized solution $U_{0}^{\mu} \in \mathcal{H}^{4}(\mathbb{R})$ is orbitally stable. For $\mu<\mu_{c}$, we have already seen that the trivial solution $a_{0}=0$ is asymptotically stable for the full neural field equation (2.1). The bifurcation at $\mu=\mu_{c}$ is subcritical for the reduced system (3.2) such that the two branches of homoclinic orbits are then orbitally unstable for the full neural field equation (2.1). It follows that these two branches are oriented backward. Let us follow these branches of solutions by decreasing values of $\mu$. We introduce the following constant:

$$
\mu_{L}=\frac{1}{\sup _{x \in \mathbb{R}}\left|S_{0}^{\prime}(x)\right| \widehat{w}_{c}} .
$$

An open question is to know if there exists or not a turning point $\left.\mu_{T} \in\right] \mu_{L}, \mu_{c}$ [ for each branch of solutions, denoted $\mu_{T_{u}}$ for the up branch and $\mu_{T_{d}}$ for the down one. At these turning points, there should be an exchange of stability and the branches should gain stability for $\mu>\mu_{T_{s}}$, $s \in\{u, d\}$. This is illustrated in Figure 2. Unfortunalety, we can only conjecture the existence of such points. Nevertheless our numerical simulations support this scenario (see 5. A rigorous proof of the existence of orbitally stable localized solutions is a challenging problem.

\subsection{Stability result with respect to the connectivity function}

The aim of the end of this section is to see under which conditions an orbitally stable localized solution $U_{0}^{\mu} \in \mathcal{H}^{4}(\mathbb{R})$ of equation $(2.1)$ is transformed when we perturb the connectivity function $w$. We define a new connectivity function $w^{\epsilon}$ for all $\epsilon \geq 0$ as

$$
w^{\epsilon}(x)=b_{1} e^{-\sigma_{1}\left((1-\epsilon)|x|+\epsilon x^{2}\right)}-b_{2} e^{-\sigma_{2}\left((1-\epsilon)|x|+\epsilon x^{2}\right)},
$$

such that $w^{\epsilon}$ is a homotopy from $w$ to a difference of Gaussian functions.

Theorem 4.3. Suppose that $U_{0}^{\mu} \in \mathcal{H}^{4}(\mathbb{R})$ is a solution of $(2.1)$ and that the linearized operator $\left.D_{Y} \mathcal{G}(Y, \mu)\right|_{Y=0}$ is invertible for a given fixed $\left.\mu \in\right] \mu_{L}, \mu_{c}[$. Then, there exists $\eta>0$ such that for all $\epsilon \in] 0, \eta\left[\right.$ there exists a unique solution $U_{\epsilon}^{\mu} \in L^{2}(\mathbb{R})$ solution of

$$
U_{\epsilon}^{\mu}(x)=\int_{\mathbb{R}} w^{\epsilon}(x-y) S_{0}\left(\mu U_{\epsilon}^{\mu}(y)\right) d y .
$$




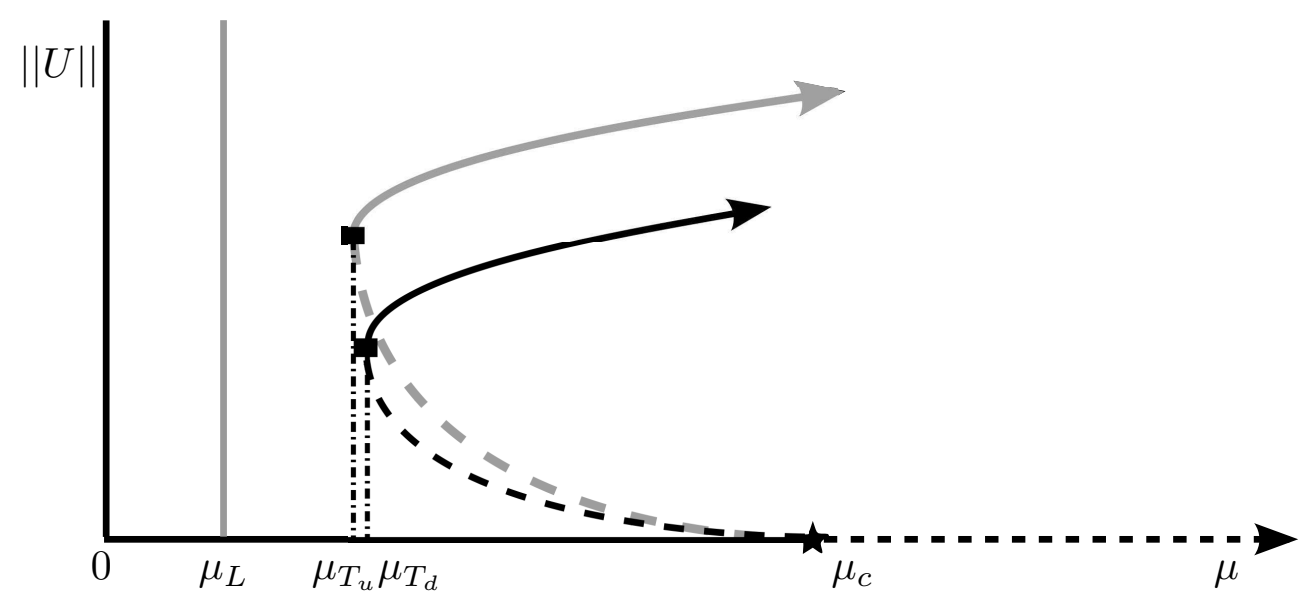

Figure 2: Bifurcation diagram for branches of localized stationary solutions of (2.1) with their stability. Solid (dashed) lines indicates stable (unstable) states. Grey (black) indicates the down (up) branch.

In particular, if $U_{0}^{\mu}$ is orbitally stable than $U_{\epsilon}^{\mu}$ is also orbitally stable.

Proof. 1. We look for solution of the form $U_{\epsilon}^{\mu}(x)=\mathcal{T}_{\rho_{\epsilon}}\left(U_{0}^{\mu}(x)+Y(x)\right)$, with $Y \in \mathcal{N}$ and $\rho_{\epsilon} \in \mathbb{R}$. Replacing $U_{\epsilon}^{\mu}$ into equation 4.13) and thanks to the translation invariance we obtain:

$$
U_{0}^{\mu}+Y=w^{\epsilon} * S\left(\mu\left(U_{0}^{\mu}+Y\right)\right)
$$

Projecting onto $\mathcal{N}$ we have:

$$
0=\mathcal{F}_{\mu}\left(U_{0}^{\mu}+Y, \epsilon\right),
$$

where $\mathcal{F}_{\mu}(U, \epsilon)=(I d-\mathbf{P})\left(-U+w^{\epsilon} * S(\mu U)\right)$.

2. We now show that $\epsilon \rightarrow \int_{\mathbb{R}} w^{\epsilon}(x-y) S(\mu U(y)) d y$ is $\mathcal{C}^{1}$ on $\mathbb{R}^{+}$.

- $y \rightarrow w^{\epsilon}(x-y) S(\mu U(y)) \in \mathrm{L}^{1}(\mathbb{R})$,

- $\epsilon \rightarrow w^{\epsilon}(x-y) S(\mu U(y)) \in \mathcal{C}^{1}\left(\mathbb{R}^{+}\right)$,

- For all $K>0$ and all $\epsilon \in[0, K]$ we have that:

$$
\left|\partial_{\epsilon}\left(w^{\epsilon}(x)\right)\right| \leq g(x) \in \mathrm{L}^{1}(\mathbb{R})
$$

with

$$
g(x)=\left\{\begin{array}{l}
\left|x^{2}-\right| x|| e^{-x} \quad x \geq 1 \\
\left|x^{2}-\right| x|| e^{-x \frac{K}{4}} \quad x \leq 1 .
\end{array}\right.
$$

Then form the theorem of differentiation under the integral sign, we have the result.

3. Equation (4.14) is an implicit equation. From the regularity of the sigmoidal function, it is clear that $y \rightarrow \mathcal{F}_{\mu}\left(U_{0}^{\mu}+Y, \epsilon\right)$ is $\mathcal{C}^{1}$ on $\mathcal{N}$. Furthermore, we have $\mathcal{F}_{\mu}\left(U_{0}^{\mu}, 0\right)=0$ and

$$
\left.D_{Y} \mathcal{F}_{\mu}\left(U_{0}^{\mu}+Y, \epsilon\right)\right|_{Y=0, \epsilon=0}=\left.D_{Y} \mathcal{G}(Y, \mu)\right|_{Y=0}
$$

From our hypothesis, we have that $\left.D_{Y} \mathcal{F}_{\mu}\left(U_{0}^{\mu}+Y, \epsilon\right)\right|_{Y=0, \epsilon=0}$ is an invertible operator from $\mathcal{N}$ to $\mathcal{N}$. 
4. We can apply the implicit function theorem which says that there exists $\eta>0$ such that for all $\epsilon \in] 0, \eta\left[, \epsilon \rightarrow Y_{\epsilon} \in \mathcal{N}\right.$ is solution of (4.14). Then, $U_{\epsilon}^{\mu}=\mathcal{T}_{\rho_{\epsilon}}\left(U_{0}^{\mu}+Y_{\epsilon}\right)$ is a solution of 4.12 for all $\rho_{\epsilon} \in \mathbb{R}$.

\section{$5 \quad$ Numerical results}

\subsection{Tuning the parameters}

Before studying localised solutions in the model it is first necessary to identify parameter ranges for which they exist. The sign of $c_{3}^{0}$ in the normal form equation 3.6 governs the existence of localised solutions as discussed in section 3.3 . The coefficient $c_{3}^{0}$ depends upon the connectivity function parameters $\left(b_{1}, b_{2}, \sigma_{1}, \sigma_{2}\right)$ and the threshold of the firing rate function $\theta$. We now describe a reduction the set of parameters governing the shape of the connectivity function. Firstly, space can be rescaled such that, without loss of generality, $\sigma_{1}=1$. In our bifurcation analysis, we have seen that the important quantities for the connectivity function are $\widehat{w}_{0}, \widehat{w}_{c}$ and $\xi_{c}$, which determine the overall shape of the Fourier transform of $w$. In order to fix the period of the critical modes bifurcating from the trivial state $a_{0}=0$ at $\mu=\mu_{c}$ to $2 * \pi$, we imposed that $\xi_{c}=1$ in Hypothesis 2, see subsection 2.2. Furthermore, the connectivity function can be reparameterized in terms of $\left(\widehat{w}_{0}, \widehat{w}_{c}\right)$ by means of the following transformation:

$$
\begin{aligned}
\sigma_{2} & =\sqrt{\frac{\widehat{w}_{c}}{\widehat{w}_{c}-\widehat{w}_{0}}}, \\
b_{1} & =-\frac{2 \widehat{w}_{c}\left(\widehat{w}_{c}-\widehat{w}_{0}\right)}{\widehat{w}_{0}}, \\
b_{2} & =-\frac{\left(2 \widehat{w}_{c}-\widehat{w}_{0}\right)^{2}}{2 \widehat{w}_{0}} \sqrt{\frac{\widehat{w}_{c}}{\widehat{w}_{c}-\widehat{w}_{0}}} .
\end{aligned}
$$

Finally, in order to express the connectivity function in terms of a single parameter, we fix $\widehat{w}_{0}=-1$. Recall that $\widehat{w}_{0}$ has to be negative in order to ensure the existence of a unique trivial solution $a_{0}$ of equation (2.1). The connectivity function only depends upon $\widehat{w}_{c}$ and can be written:

$$
w(x)=2 \widehat{w}_{c}\left(\widehat{w}_{c}+1\right) e^{-|x|}-\frac{\left(2 \widehat{w}_{c}+1\right)^{2}}{2} \sqrt{\frac{\widehat{w}_{c}}{\widehat{w}_{c}+1}} e^{-\sqrt{\frac{\widehat{w}_{c}}{w_{c}+1}}|x|} .
$$

It follows directly from the above discussion that, defined in this way, $w$ satisfies the conditions $\widehat{w}_{0}=-1$ and $\xi_{c}=1$.

We plot the contour $c_{3}^{0}=0$ as a function of $\left(\widehat{w}_{c}, \theta\right)$ in Figure 3 . We can see that there exists a non empty region of the parameters where the condition $c_{3}^{0}<0$ is satisfied; therefore, there exist branches of spatially localized solutions within this region. For small values of $\theta c_{3}^{0}>0$ and spatially localized solutions do not exist.

\subsection{Numerical computation of the stability of localized solutions}

In order to numerically investigate the stability of a localized solution $U_{0}^{\mu}$ of equation (2.1), we have to solve the following eigenvalue problem. We start from equation (4.1) with $p \in \mathcal{H}^{4}(\mathbb{R})$ and apply the PDE method developed in 3.1. We obtain:

$$
(\sigma+1) \mathcal{L}_{0}(p(x))=\mu\left(\Gamma_{1}-\Gamma_{2} \partial_{x^{2}}^{2}\right)\left[S_{0}^{\prime}\left(\mu U_{0}^{\mu}(x) p(x)\right]\right.
$$






Figure 3: Coefficient $c_{3}^{0}$ and connectivity function. (a) Shows the curve $c_{3}^{0}=0$ in the $\left(\theta, \widehat{w}_{c}\right)$ plane; for values to the right of this curve $c_{3}^{0}<0$. Inset (a1) shows the wizard hat connectivity function $w$ and its Fourier transform $\widehat{w}$ for $\widehat{w}_{c}=5$.

where $\mathcal{L}_{0}=\partial_{x^{4}}^{4}-\left(\sigma_{1}^{2}+\sigma_{2}^{2}\right) \partial_{x^{2}}^{2}+\sigma_{1}^{2} \sigma_{2}^{2}$. For all $p \in \mathcal{H}^{4}(\mathbb{R})$ and $q \in \mathrm{L}^{2}(\mathbb{R})$, we set $\mathcal{L}_{0} p=q$. As the spectrum of $\mathcal{L}_{0}$ is given by $\operatorname{spec}_{\mathcal{L}_{0}}=\left\{\xi^{4}+\left(\sigma_{1}^{2}+\sigma_{2}^{2}\right) \xi^{2}+\sigma_{1}^{2} \sigma_{2}^{2} \mid \xi \in \mathbb{R}\right\} \subset\left[\sigma_{1}^{2} \sigma_{2}^{2},+\infty\left[, \mathcal{L}_{0}\right.\right.$ is an invertible operator and $p=\mathcal{L}_{0}^{-1} q$. It follows that equation 5.1 can be rewritten:

$$
\sigma q(x)=-q(x)+\mu\left(\Gamma_{1}-\Gamma_{2} \partial_{x^{2}}^{2}\right)\left[S_{0}^{\prime}\left(\mu U_{0}^{\mu}(x) \mathcal{L}_{0}^{-1} q(x)\right]=\mathcal{B}(q(x)) .\right.
$$

Now, for every solution $U_{0}^{\mu}$ discretized on a domain $[-L, 0]$, we compute the eigenvalues $\sigma$ of the corresponding discretized version of the linear operator $\mathcal{B}$ where we use finite differences methods to approximate the Laplacian operator $\partial_{x^{2}}^{2}$. As we numerically work on a finite domain, the 0 eigenvalue due to translation invariance in the full model is not present in the spectrum of the discretizated version of the operator $\mathcal{B}$. When all eigenvalues have negative real part, then the solution is orbitally stable, otherwise it is unstable.

\subsection{Snaking behaviour and localized solutions varying $\mu$}

In this section we use the numerical continuation package AUTO [14] with the extension HOMCONT to compute homoclinic solutions of the system of ODEs described by (3.3). Solutions of this system corresponds to steady states of the neural field equation (2.1) where the spatial coordinate $x$ has been recast as the time variable in the ODE system.

Starting data for the continuation analysis is obtained by solving the system (3.3) on the halfinterval $x \in[-L, 0]$ with the four boundary conditions $u_{1}(-L)=u_{3}(-L)=u_{1}(0)=u_{3}(0)=0$; a boundary value problem (BVP) solver in the software package Matlab was used. Based on the analytical results presented in this paper, for fixed $\left(\widehat{w}_{c}, \theta\right)$ we set $\mu$ to a value less than but still close to $\mu_{c}$, where two types of unstable localized solutions are known to exist as discussed in subsection 3.4. Up solutions, for which $u_{1}(0)>0$, and down solutions, for which $u_{1}(0)<0$, are found by providing different initial conditions to the BVP solver. Using these solutions as starting data in AUTO, branches of localized solutions were tracked under the variation of $\mu$.

Figure 4 shows a bifurcation diagram in the parameter $\mu$ that gives an exact qualitative agreement with the analytical results as summarised in Figure 2 A branch of trivial solutions $(\|U\|=0)$ is stable for $\mu<\mu_{c}$ and unstable after undergoing a reversible Hopf bifurcation $R H_{\mu_{c}}$ at $\mu=\mu_{c}$. There are two subcritical, unstable bifurcating branches, one corresponding to the up solution and one corresponding to the down solution. The up and down branches undergo fold bifurcations at $F_{u l}^{1}$ and $F_{d l}^{1}$ in which the branches gain stability as predicted analytically. 

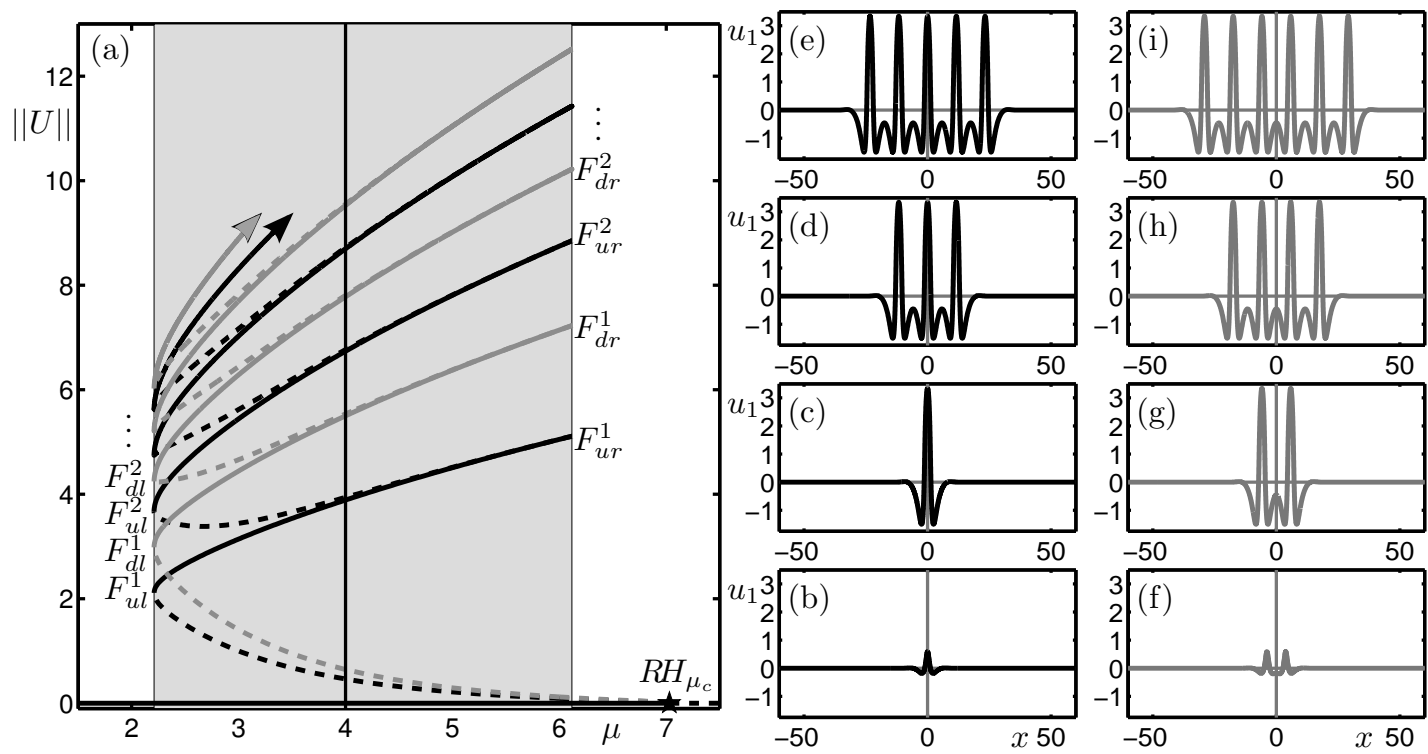

Figure 4: Snaking behaviour and localized solutions at $\widehat{w}_{c}=5$ and $\theta=3.5$. (a) Bifurcation diagram in $\mu$ where stable branches are solid curves and unstable branches are dashed curves. A trivial branch of solutions undergoes a reversible Hopf bifurcation at $R H_{\mu_{c}}$. Bifurcating branches corresponding to up solutions are black and to down solutions are grey. These branches undergo a series of fold bifurcations that bound a $\mu$-range (shaded gray) in which localized solutions exist; see text. A vertical gray line corresponds to $\mu=4$. (b) Solution profile at $\mu=4$ on the lowest unstable up solution branch. (c)-(e) Solution profiles at $\mu=4$ on the subsequent stable up solution branch segments. (f) Solution profile at $\mu=4$ on the lowest unstable down solution branch. (g)-(i) Solution profiles at $\mu=4$ on the subsequent stable down solution branch segments. 

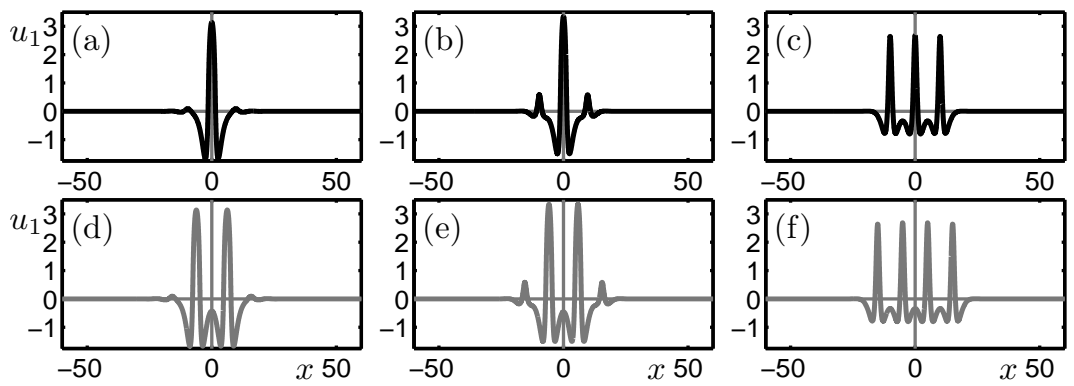

Figure 5: Adding of a bump through fold bifurcations. (a) Solution profile at $F_{u r}^{1}$. (b) Solution profile at $\mu=4$ on unstable branch between $F_{u r}^{1}$ and $F_{u l}^{2}$. (c) Solution profile at $F_{u l}^{2}$. (a) Solution profile at $F_{d r}^{1}$. (b) Solution profile at $\mu=4$ on unstable branch between $F_{d r}^{1}$ and $F_{d l}^{2}$. (c) Solution profile at $F_{d l}^{2}$.

A series of fold bifurcations $F_{*}^{n}$ bound a region in which stable localized solutions persist, with additional bumps added with increasing $n$, moving up the diagram. In the subscript notation for the folds $u$ and $d$ correspond to up and down solutions, whereas $l$ and $r$ correspond to the left and right boundaries of the region in which stable localized solutions persist.

The panels (b)-(i) in Figure 4 show the solution profiles $u_{1}$ of the homoclinic cycles on the full interval $x \in[-L, L]$ by taking into account the reflectional symmetry about $x=0$. All panels correspond to solutions at $\mu=4$. The bottom panels (b) and (f) are from the lowest unstable up and down branches, respectively. Subsequent panels show solutions from the stable branch segments only; for example, panel (c) from the stable up branch between $F_{u l}^{1}$ and $F_{u r}^{1}$, panel (g) from the stable down branch between $F_{d l}^{1}$ and $F_{d r}^{1}$, and so on. In general, for the up case there are $2 n-1$ bumps on the stable branch between $F_{u l}^{n}$ and $F_{u r}^{n}$ and for the down case there are $2 n$ bumps on the stable branch between $F_{d l}^{n}$ and $F_{d r}^{n}$. The computations terminate (arrows in panel (a)) at $n=4$; beyond this the model will no longer be valid when the localized solutions approach the limits of finite domain at $x= \pm L$.

In order to illustrate the way in which bumps are added we show in Figure 5 solution profiles at the right-hand folds for $n=1$, at the left-hand folds for $n=2$ and at the intermediate value of $\mu=4$ on the unstable branches connecting these fold points. Panels (a)-(c) show the up case and panels (d)-(f) show the down case. One can see in panels (a) and (d) that at the right-hand fold points $\left(F_{u r}^{1}\right.$ and $F_{d r}^{1}$ ) the new bump first appears; the bumps gradually grow along the unstable branch between the fold points as shown in panels (b) and (e) and are finally the same size as the existing bumps at the left-hand fold point $\left(F_{u l}^{2}\right.$ and $\left.F_{d l}^{2}\right)$, as shown in panels (c) and (f). Note that with decreasing $\mu$ the overall range in $u_{1}$ of the solutions decreases approaching the left-hand fold points $\left(F_{u l}^{2}\right.$ and $\left.F_{d l}^{2}\right)$, but increases again on the stable branch; compare Figure 5 (a) with Figure $4(\mathrm{~d})$ and Figure $5(\mathrm{e})$ with Figure $4(\mathrm{~h})$. The sequence described is analagous for the addition of further bumps.

\subsection{Regions of localized solutions in the parameter plane}

In the previous section, for specific values of the critical Fourier mode $\widehat{w}_{c}=5$ and the threshold $\theta=3.5$, we identified a particular range of $\mu$ for which localized solutions exist. The aim here is to show that localized solutions exist over a range of the other system parameters and are not an isolated phenomena in parameter space. Further, we aim to identify exactly the ranges of the three parameters $\mu, \widehat{w}_{c}$ and $\theta$ for which localized solutions perist. First we observe that, as 

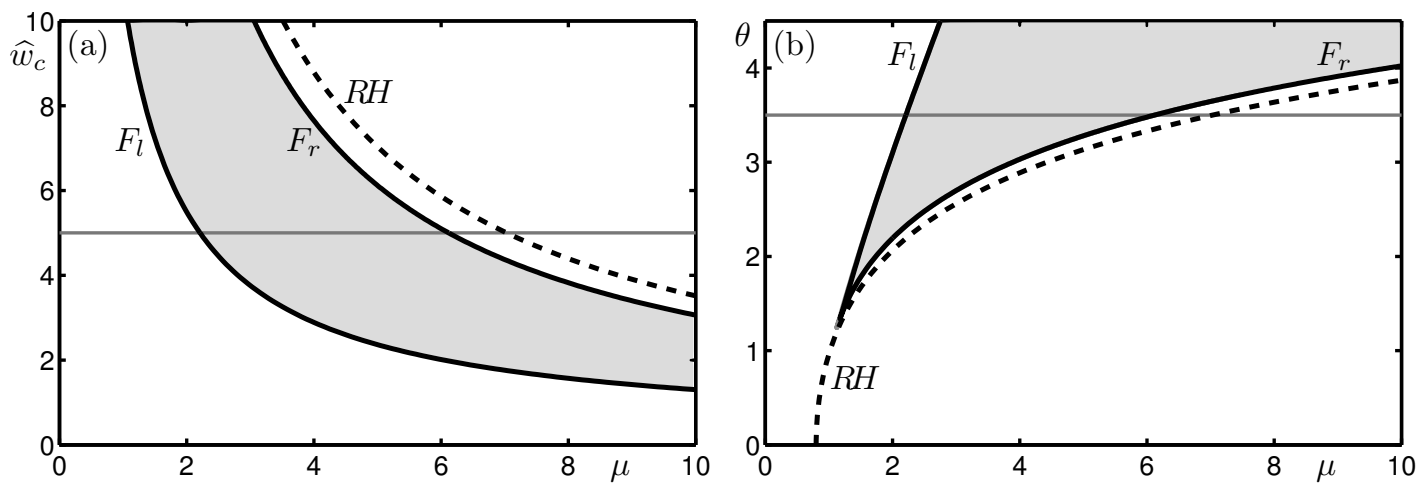

Figure 6: Two-parameter bifurcation diagrams. (a) Bifurcation curves in the $\left(\mu, \widehat{w}_{c}\right)$-plane. The locus of the reversible Hopf bifurcation $R H$ is a dashed black curve. The loci of the left-hand $F_{l}$ and right-hand $F_{r}$ fold bifurcations in the snaking structure shown in Figure 4 are solid black curves. A horizontal gray line corresponds with $\widehat{w}_{c}=5$, the value used in Figure 4. (b) Similarly indicated bifurcation curves plotted in the $(\mu, \theta)$-plane. The fold curves $F_{l}$ and $F_{r}$ are connected at a cusp point. A horizontal gray line corresponds with $\theta=3.5$ the value used in Figure 4 .

shown in Figure 4(a), all the $\mu$-values associated with the fold points for both the up and down solutions are aligned on the left and right boundaries as demarkated by the gray shaded region. Therefore, assuming that this is also the case when $\widehat{w}_{c}$ and $\theta$ are varied, we can find bounds of regions for which localized solutions exist; for example, in the $\left(\mu, \widehat{w}_{c}\right)$-plane it is sufficient to track the loci of the fold points $F_{u l}^{1}$ and $F_{u r}^{1}$ under the simultaneous variation of those two parameters. We denote the coinciding fold points at the left-hand boundary as $F_{l}$ and the coinciding fold points at the right-hand boundary $F_{r}$.

Figure 6(a) and (b) show curves that are the loci of bifurcations in the $\left(\mu, \widehat{w}_{c}\right)$ and $(\mu, \theta)$ parameter planes, respectively. In each panel, the curve $R H$ is the locus of the reversible hopf bifurcation at $\mu_{c}$, which is determined analytically by the expression $\mu_{c}=\left(s_{1} \widehat{w}_{c}\right)^{-1}$. The curves $F_{l}$ and $F_{r}$ bound the region for which localized solutions persist (shaded in gray). A horizontal line in each panel corresponds to the default values of $\widehat{w}_{c}$ and $\theta$ used in Figure 4 . Under the variation of $\mu$ and $\widehat{w}_{c}$, shown in Figure 6(a), there is a channel in parameter space for which localized solutions exist; as $\widehat{w}_{c}$ is increased the channel becomes narrower in $\mu$ and shifts to lower values of $\mu$; as $\widehat{w}_{c}$ is decreased the channel becomes wider in $\mu$ and shifts to higher values of $\mu$. Under the variation of $\mu$ and $\theta$ as shown in Figure 6(b) the range of $\mu$ for localized solutions grows and shifts to the right with increasing $\theta$. For decreasing $\theta$ the $\mu$ range decreases and contracts to a cusp point that is effectively lower bound on $\theta$, below which there are no localized solutions. We note that in both cases the localized solutions always exist before the reversible Hopf. Therefore, the localized solutions coexist with a stable trivial solution.

In [11, the authors present a snaking diagram computed under the variation of the threshold of the sigmoidal function $\theta$; in their formalism the sigmoid slope is $\mu=30$. Their connectivity function was taken to be $w(x)=\frac{w_{0}}{4}(1-|x|) e^{-|x|}$ with Fourier transform $\widehat{w}(\xi)=w_{0} \frac{\xi^{2}}{\left(1+\xi^{2}\right)^{2}}$, which gives $\widehat{w}_{0}=0, \xi_{c}=1$ and $\widehat{w}_{c}=\frac{w_{0}}{4}>0$. In Figure 6(b) we recover their result: for a fixed value of $\mu$ and $\widehat{w}_{c}$, increasing the threshold leads to a supercritical reversible Hopf bifurcation. Note that, even though the bifurcation is supercritical, the trivial solution is unstable before the bifurcation and gains stability at the $R H$ point. In [26, 15] the authors presented a study of snaking-type behaviour; however, as was noted in [26], the normal form theory that we have developed in this 
article is not applicable in their formalism, such that it is difficult to characterize their results from a bifurcation point of view. The shape of the snaking diagrams plotted in [26, 15] shows that, when increasing the parameter $b$ in equation 1.3 (the equivalent of $\widehat{w}_{c}$ in our setting), branches of localized solutions have the opposite orientation to Figure 4(a). This suggests a supercritical type of bifurcation in $b$; we conjecture that the difference in behaviour is mainly due to the shape of nonlinearity $S$ which is not analytical at the trivial solution in [26, 15].

\section{Discussion}

In this article we have presented a neural field equation set on the real line with a "wizard hat" connectivity function and an analytical firing rate function. Applying successively a Fourier transform and then an inverse Fourier transform to our system, we have been able to transform our initial integro-differential equation into a partial differential equation involving spatial derivatives of even order. Time-independant spatially localized solutions of our problem satisfy a fourth order reversible dynamical system in the space variable. For some critical value of the slope of the firing rate function, a Hopf bifurcation with resonance occurs from the trivial state. We have computed the coefficients of the normal form for the 1:1 reversible Hopf bifurcation. This has allowed us to find sufficient conditions on the parameters of our model for the existence of homoclinic orbits to the trivial states and thus spatially localized states. We have also shown that our results extend to other types of connectivity function for which the PDE method can not been applied (difference of Gaussian functions for example).

Numerical continuation was used to follow branches of homoclinic cycles corresponding to localized states and, thus, confirm the description of the system's solution structure both close to the reversible Hopf bifurcation and on the associated bifurcated branches (as described by the analytical results presented herein). Further, varying the sigmoid slope, we show that the system exhibits snaking behaviour: a series fold bifurcations accumulating at lower and upper limits bound a parameter range over which stable localized solutions perist. The loci of these fold bifurcations, which can be followed using two-parameter continuation, form the boundaries of regions of localized solutions in the parameter plane. Importantly, we show that localized solutions are not an isolated phenomenon in parameter space, that they exist over wide ranges of three parameters and that it is possible to produce a snaking diagram in any of these parameters. Another important result is that, for small values of the threshlod of the sigmoid function, there are no localized solutions.

We think that this work is a first step towards a rigorous study of stationary radially symmetric solutions of neural field equations set on the Euclidean plane with smooth firing rate function. To our knowledge, only numerical analyses have been done so far [25, 27, 13, 12, 31 , with Heaviside-like firing rate function. We propose that the methods developed in [28, 29] for the construction of radial localized solutions of Swift-Hohenberg equation on the Euclidean plane, would be equally applicable to the neural field equation using the results presented in this article as a strating point. Another interesting avenue for future work will be to study radially symmetric solutions for a neural field model of texture perception in visual areas [9, 19, 8, 18, which is set on the Poincaré disk.

Acknowledgements The authors are grateful to David Lloyd for his helpful comments on the preparation of the present manuscript. The authors would also like to thank Bart Oldeman for his help with the functionality of AUTO. This work was partially funded by the ERC advanced grant NerVi number 227747. 


\section{A Proof of Lemma 2.1}

We recall the Lemma 2.1 stated in subsection 2.2 .

Lemma A.1. There exists a unique solution $\left(\mu_{c}, a_{c}=a_{0}\left(\mu_{c}\right)\right)$ of:

$$
\left\{\begin{array}{l}
a_{c}=\widehat{w}_{0} S\left(\mu_{c} a_{c}\right) \\
1=\mu_{c} S^{\prime}\left(\mu_{c} a_{c}\right) \widehat{w}_{c} .
\end{array}\right.
$$

It is possible to express $\left(\mu_{c}, a_{c}\right)$ with the following analytic formulas:

$$
\begin{aligned}
\mu_{c} & =\frac{\widehat{w}_{0}^{2}}{a_{c} \widehat{w}_{c}\left(\widehat{w}_{0}-a_{c}\right)}, \\
a_{c} & =\frac{\widehat{w}_{0} \widehat{w}_{c} W\left(-\widehat{w}_{0} e^{-\frac{-\widehat{w}_{0}+\theta \widehat{w}_{c}}{\widehat{w}_{c}}} / \widehat{w}_{c}\right)}{\widehat{w}_{c} W\left(-\widehat{w}_{0} e^{-\frac{-\widehat{w}_{0}+\theta \widehat{w}_{c}}{\hat{w}_{c}}} / \widehat{w}_{c}\right)-\widehat{w}_{0}} .
\end{aligned}
$$

$W$ is the Lambert function which satisfies $W(x) e^{W(x)}=x$.

Proof. It has been shown in 33 that if $\widehat{w}_{0}<0$ then system A.1) has a unique solution $\left(\mu_{c}, a_{c}=\right.$ $\left.a_{0}\left(\mu_{c}\right)\right)$. Formulas for $a_{c}$ and $\mu_{c}$ are related to the properties of the sigmoidal function $S$ defined in equation 2.2 . Using the fact that $S$ satisfies the ordinary differential equations $S^{\prime}=S(1-S)$ and combining the two equations of (A.1), we find:

$$
\mu_{c}=\frac{\widehat{w}_{0}^{2}}{a_{c} \widehat{w}_{c}\left(\widehat{w}_{0}-a_{c}\right)} .
$$

If $m=\frac{\widehat{w}_{c}}{\widehat{w}_{0}}$ and $x=a_{c} / \widehat{w}_{0}$, from $a_{c}=\widehat{w}_{0} S\left(\mu_{c} a_{c}\right), x$ is solution of the equation:

$$
\begin{gathered}
\frac{1}{x}=1+\exp \left(-\frac{1}{m(1-x)}+\theta\right) \\
\Leftrightarrow x=(1-x) \exp \left(+\frac{1}{m(1-x)}-\theta\right) \\
\Leftrightarrow \frac{x}{m(x-1)} \exp \left(\frac{x}{m(x-1)}\right)=-\frac{1}{m} \exp \left(-\frac{-1+\theta m}{m}\right) \\
\Leftrightarrow \frac{x}{m(x-1)}=W\left(-\frac{1}{m} \exp \left(-\frac{-1+\theta m}{m}\right)\right) \\
\Leftrightarrow x=\frac{m W\left(-\frac{1}{m} \exp \left(-\frac{-1+\theta m}{m}\right)\right)}{m W\left(-\frac{1}{m} \exp \left(-\frac{-1+\theta m}{m}\right)\right)-1}
\end{gathered}
$$

which gives the desired formula.

\section{B Proof of Lemma 2.2}

This section is devoted to the proof of Lemma 2.2 .

$\mathrm{RR} \mathrm{n}^{\circ} 7872$ 


\section{B.1 Computation of $\nu$}

We recall that the linear operator $\mathbf{L}_{\mu}$ is given by:

$$
\mathbf{L}_{\mu} v=-v+\mu s_{1} w * v .
$$

It is straightforward to see that $\mathbf{L}_{\mu} e^{i x}=\left(-1+\mu s_{1} \widehat{w}_{c}\right) e^{i x}=\frac{\lambda}{\mu_{c}} e^{i x}$. As a consequence $\nu=\frac{1}{\mu_{c}}$.

\section{B.2 Computation of $\chi$}

We have defined $\mathbf{R}$ as the following nonlinear operator:

$$
\mathbf{R}(v, \lambda)=w * f_{0}(v, \lambda) .
$$

We can Taylor expand $\mathbf{R}$ at $(v=0, \lambda=0)$ and if we denote:

$$
\begin{aligned}
\mathbf{R}_{11}(v, \lambda) & =\lambda s_{1} w * v \\
\mathbf{R}_{20}(v, w) & =\frac{\mu_{c}^{2} s_{2}}{2} w *(v w), \\
\mathbf{R}_{30}(u, v, w) & =\frac{\mu_{c}^{3} s_{3}}{6} w *(u v w),
\end{aligned}
$$

then, we find that $\mathbf{R}(v, \lambda)=\mathbf{R}_{11}(v, \lambda)+\mathbf{R}_{20}(v, v)+\mathbf{R}_{30}(v, v, v)+$ h.o.t. We also Taylor expand $\Phi:$

$$
\Phi(Z, \bar{Z}, \lambda)=\sum_{s, l, m} Z^{s} \bar{Z}^{l} \lambda^{m} \Phi_{s l m}
$$

Applying classical techniques [10, 22] we obtain the following equations:

$$
\begin{aligned}
0 & =\mathbf{L}_{\mu_{c}} \Phi_{200}+\mathbf{R}_{20}\left(e^{i x}, e^{i x}\right) \\
0 & =\mathbf{L}_{\mu_{c}} \Phi_{110}+2 \mathbf{R}_{20}\left(e^{i x}, e^{-i x}\right) \\
\chi & =\left\langle 2 \mathbf{R}_{20}\left(e^{-i x}, \Phi_{200}\right)+2 \mathbf{R}_{20}\left(e^{i x}, \Phi_{110}\right)+3 \mathbf{R}_{30}\left(e^{i x}, e^{i x}, e^{-i x}\right), e^{i x}\right\rangle_{\mathrm{L}_{p e r}^{2}[0,2 \pi]} .
\end{aligned}
$$

If we denote $\widehat{w}_{2}=\widehat{w}(2)$, the two first equations are solved with:

$$
\begin{aligned}
& \Phi_{200}=\mu_{c}^{2} s_{2} \frac{\widehat{w}_{2}}{2\left(1-\widehat{w}_{2} / \widehat{w}_{c}\right)} e^{2 i x}+\operatorname{Span}\left(e^{i x}, e^{-i x}\right) \\
& \Phi_{110}=\mu_{c}^{2} s_{2} \frac{\widehat{w}_{0}}{1-\widehat{w}_{0} / \widehat{w}_{c}}+\operatorname{Span}\left(e^{i x}, e^{-i x}\right) .
\end{aligned}
$$

It follows that $\chi$ is given by:

$$
\chi=\mu_{c}^{3} \widehat{w}_{c}\left[\frac{s_{3}}{2}+\mu_{c} s_{2}^{2}\left(\frac{\widehat{w}_{2}}{2\left(1-\widehat{w}_{2} / \widehat{w}_{c}\right)}+\frac{\widehat{w}_{0}}{1-\widehat{w}_{0} / \widehat{w}_{c}}\right)\right] .
$$

Relations 2.10 give the formula for $\chi$, as stated in the Lemma.

\section{Proof of Lemma 3.2}

This section is devoted to the proof of Lemma 3.2. Firstly, the four eigenvalues of $D_{0} \mathbf{F}$, when $\lambda>0$, are given by:

$$
X= \pm i\left(1 \pm \sqrt{\frac{\lambda s_{1}\left(\Gamma_{1}+\Gamma_{2}\right)}{4}}+\frac{\lambda s_{1}\left(\Gamma_{2}-\Gamma_{1}\right)}{8}+0\left(|\lambda|^{3 / 2}\right)\right) .
$$


We observe that the eigenvalues of the linearization at the origin of the normal form 3.6 are given by:

$$
i\left(1 \pm \sqrt{-c_{1}^{1} \lambda}+\alpha \lambda+0\left(|\lambda|^{3 / 2}\right)\right)
$$

where $c_{1}^{1}$ and $\alpha$ are the coefficients in the expensions of $P$ and $Q$ which implies that:

$$
c_{1}^{1}=-\frac{s_{1}\left(\Gamma_{1}+\Gamma_{2}\right)}{4}, \quad \alpha=\frac{s_{1}\left(\Gamma_{2}-\Gamma_{1}\right)}{8} .
$$

It remains to compute the coefficients $c_{3}^{0}, \beta, c, \gamma$ of the expansions $P$ and $Q$ which requires the Taylor expansion of $\mathbf{F}$. First of all, we have for the nonlinearity defined in equation $(3.4)$ :

$$
\mathcal{R}(U, \lambda)=\mathcal{R}_{1,1}(U, \lambda)+\mathcal{R}_{2,0}(U, U)+\mathcal{R}_{3,0}(U, U, U)+\text { h.o.t. }
$$

and if $U=\left(u_{1}, u_{2}, u_{3}, u_{4}\right), V=\left(v_{1}, v_{2}, v_{3}, v_{4}\right)$ and $W=\left(w_{1}, w_{2}, w_{3}, w_{4}\right)$ we have:

$$
\begin{aligned}
\mathcal{R}_{2,0}(U, V)= & \frac{\mu_{c}^{2} s_{2}}{2}\left(0,0,0, \Gamma_{1} u_{1} v_{1}-\Gamma_{2}\left(2 u_{2} v_{2}+u_{1} v_{3}+u_{3} v_{1}\right)\right)^{\mathbf{T}} \\
\mathcal{R}_{3,0}(U, V, W)= & \frac{\mu_{c}^{3} s_{3}}{6}\left(0,0,0, \Gamma_{1} u_{1} v_{1} w_{1}-\Gamma_{2}\left(u_{1} v_{1} w_{3}+u_{1} w_{1} v_{3}\right.\right. \\
& \left.\left.+u_{3} v_{1} w_{1}+2\left(u_{1} v_{2} w_{2}+u_{2} v_{1} w_{2}+u_{2} v_{2} w_{1}\right)\right)\right)^{\mathbf{T}}
\end{aligned}
$$

The Taylor expansion of $\Psi$ in the normal form 3.6 is given by:

$$
\Psi(A, B, \bar{A}, \bar{B}, \lambda)=\sum_{1 \leq r+s+q+l+m \leq p} A^{r} B^{s} \bar{A}^{q} \bar{B}^{l} \lambda^{m} \Psi_{r s q l m} .
$$

Using the expansions of $\mathcal{R}, \Psi, P$ and $Q$, we end up with equations at different orders.

\section{C.1 Computation of $c_{3}^{0}$}

Applying the method describes in the appendix of Haragus-Iooss [22, we have first to solve the two following equations:

$$
\begin{aligned}
& \mathcal{O}\left(A^{2}\right): \quad 0=\left(\mathcal{A}-2 i \mathbb{I}_{\mathbb{R}^{4}}\right) \Psi_{20000}+\mathcal{R}_{2,0}\left(\zeta_{0}, \zeta_{0}\right) \\
& \mathcal{O}(A \bar{A}): \quad 0=\mathcal{A} \Psi_{10100}+2 \mathcal{R}_{2,0}\left(\zeta_{0}, \bar{\zeta}_{0}\right) \\
& \mathcal{O}\left(A^{2} \bar{A}\right): \quad c_{3}^{0} \zeta_{1}+i \beta \zeta_{0}=\left(\mathcal{A}-i \mathbb{I}_{\mathbb{R}^{4}}\right) \Psi_{20100}+2 \mathcal{R}_{2,0}\left(\zeta_{0}, \Psi_{10100}\right) \\
& +2 \mathcal{R}_{2,0}\left(\bar{\zeta}_{0}, \Psi_{20000}\right)+3 \mathcal{R}_{3,0}\left(\zeta_{0}, \zeta_{0}, \bar{\zeta}_{0}\right) \text {. }
\end{aligned}
$$

The invertibility of the operators $\mathcal{A}, \mathcal{A}-2 i \mathbb{I}_{\mathbb{R}^{4}}$ implies that solutions of equations (C.1) and C.2 are given by

$$
\Psi_{20000}=-\left(\mathcal{A}-2 i \mathbb{I}_{\mathbb{R}^{4}}\right)^{-1} \mathcal{R}_{2,0}\left(\zeta_{0}, \zeta_{0}\right)=\frac{\mu_{c}^{2} s_{2}\left(\left(\Gamma_{1}+4 \Gamma_{2}\right)\right.}{18}(1,2 i,-4,-8 i)^{\mathbf{T}}
$$

and as

$$
\mathcal{R}_{2,0}\left(\zeta_{0}, \bar{\zeta}_{0}\right)=\frac{\mu_{c}^{2} s_{2} \Gamma_{1}}{2}(0,0,0,1)^{\mathbf{T}}
$$

$\mathrm{RR} \mathrm{n}^{\circ} 7872$ 
we automatically have:

$$
\Psi_{10100}=-2 \mathcal{A}^{-1} \mathcal{R}_{2,0}\left(\zeta_{0}, \bar{\zeta}_{0}\right)=\mu_{c}^{2} s_{2} \Gamma_{1}(1,0,0,0)^{\mathbf{T}}
$$

In order to compute the expression of $c_{3}^{0}$ in equation (C.3), we need to define a vector orthogonal to $\left(\zeta_{0}, \bar{\zeta}_{1}, \bar{\zeta}_{0}\right)$ for the natural Hermitian scalar product $\langle\cdot, \cdot\rangle$. Let be $\zeta_{1}^{*}$ the vector in the kernel of $(\mathcal{A}-i)^{*}$,

$$
\zeta_{1}^{*}=-\frac{1}{4}(-i, 1,-i, 1)^{\mathbf{T}}
$$

which satisfies

$$
\left\langle\zeta_{1}, \zeta_{1}^{*}\right\rangle=1, \quad\left\langle\zeta_{0}, \zeta_{1}^{*}\right\rangle=0, \quad\left\langle\bar{\zeta}_{1}, \zeta_{1}^{*}\right\rangle=0, \quad\left\langle\bar{\zeta}_{0}, \zeta_{1}^{*}\right\rangle=0
$$

Taking the scalar product of equation C.3 with $\zeta_{1}^{*}$ we obtain a relation for $c_{3}^{0}$

$$
c_{3}^{0}=\left\langle 2 \mathcal{R}_{2,0}\left(\zeta_{0}, \Psi_{10100}\right)+2 \mathcal{R}_{2,0}\left(\bar{\zeta}_{0}, \Psi_{20000}\right)+3 \mathcal{R}_{3,0}\left(\zeta_{0}, \zeta_{0}, \bar{\zeta}_{0}\right), \zeta_{1}^{*}\right\rangle
$$

with

$$
\begin{aligned}
\mathcal{R}_{2,0}\left(\zeta_{0}, \Psi_{10100}\right) & =\frac{\mu_{c}^{4} s_{2}^{2} \Gamma_{1}\left(\Gamma_{1}+\Gamma_{2}\right)}{2}(0,0,0,1)^{\mathbf{T}} \\
\mathcal{R}_{2,0}\left(\bar{\zeta}_{0}, \Psi_{20000}\right) & =\frac{\mu_{c}^{4} s_{2}^{2}\left(\Gamma_{1}+\Gamma_{2}\right)\left(\Gamma_{1}+4 \Gamma_{2}\right)}{36}(0,0,0,1)^{\mathbf{T}} \\
\mathcal{R}_{3,0}\left(\zeta_{0}, \zeta_{0}, \bar{\zeta}_{0}\right) & =\frac{\mu_{c}^{3} s_{3}\left(\Gamma_{1}+\Gamma_{2}\right)}{6}(0,0,0,1)^{\mathbf{T}}
\end{aligned}
$$

We deduce that

$$
c_{3}^{0}=-\frac{\mu_{c}^{3}\left(\Gamma_{1}+\Gamma_{2}\right)}{4}\left[\frac{s_{3}}{2}+\frac{\mu_{c} s_{2}^{2}\left(19 \Gamma_{1}+4 \Gamma_{2}\right)}{18}\right]
$$

\section{C.2 Computation of $\beta$ and $c$}

If decompose $\widetilde{\Psi}_{20100}$ on $\left(\zeta_{0}, \zeta_{1}\right)$ such that

$$
\Psi_{20100}=\widetilde{\Psi}_{20100}+i \beta \zeta_{1}+\psi_{20100} \zeta_{0}, \text { with } \psi_{20100} \in \mathbb{R}
$$

then equation C.3 can be rewritten with $\widetilde{\Psi}_{20100}$ only:

$$
\begin{aligned}
c_{3}^{0} \zeta_{1}= & \left(\mathcal{A}-i \mathbb{I}_{\mathbb{R}^{4}}\right) \widetilde{\Psi}_{20100}+2 \mathcal{R}_{2,0}\left(\zeta_{0}, \Psi_{10100}\right)+2 \mathcal{R}_{2,0}\left(\bar{\zeta}_{0}, \Psi_{20000}\right) \\
& +3 \mathcal{R}_{3,0}\left(\zeta_{0}, \zeta_{0}, \bar{\zeta}_{0}\right)
\end{aligned}
$$

Knowing each terms of the previous equation allows us to calculate $\widetilde{\Psi}_{20100}$ :

$$
\widetilde{\Psi}_{20100}=c_{3}^{0}(0,0,1,3 i)^{\mathbf{T}} .
$$

The coefficients $\beta$ and $c$ are obtained from orders $\mathcal{O}\left(A^{2} \bar{B}\right)$ and $\mathcal{O}(A \bar{A} B)$ :

$$
\begin{aligned}
\frac{i c}{2} \zeta_{1}-\frac{\gamma}{2} \zeta_{0}+\Psi_{20100}= & \left(\mathcal{A}-i \mathbb{I}_{\mathbb{R}^{4}}\right) \widetilde{\Psi}_{20010}+2 \mathcal{R}_{2,0}\left(\zeta_{0}, \Psi_{10010}\right) \\
& +2 \mathcal{R}_{2,0}\left(\bar{\zeta}_{1}, \Psi_{20000}\right)+3 \mathcal{R}_{3,0}\left(\zeta_{0}, \zeta_{0}, \bar{\zeta}_{1}\right)
\end{aligned}
$$




$$
\begin{aligned}
\left(i \beta-\frac{i c}{2}\right) \zeta_{1}+\frac{\gamma}{2} \zeta_{0}+2 \Psi_{20100}= & \left(\mathcal{A}-i \mathbb{I}_{\mathbb{R}^{4}}\right) \widetilde{\Psi}_{11100}+2 \mathcal{R}_{2,0}\left(\zeta_{0}, \Psi_{01100}\right) \\
& +2 \mathcal{R}_{2,0}\left(\bar{\zeta}_{0}, \Psi_{11000}\right)+2 \mathcal{R}_{2,0}\left(\zeta_{1}, \Psi_{10100}\right) \\
& +6 \mathcal{R}_{3,0}\left(\zeta_{0}, \zeta_{1}, \bar{\zeta}_{0}\right) .
\end{aligned}
$$

Taking scalar product with $\zeta_{1}^{*}$ equations $(\mathrm{C.5})$ and $(\mathrm{C} .6)$ now give

$$
\begin{aligned}
i \beta+\frac{i c}{2}= & \left\langle 2 \mathcal{R}_{2,0}\left(\zeta_{0}, \Psi_{10010}\right)+2 \mathcal{R}_{2,0}\left(\bar{\zeta}_{1}, \Psi_{20000}\right)+3 \mathcal{R}_{3,0}\left(\zeta_{0}, \zeta_{0}, \bar{\zeta}_{1}\right), \zeta_{1}^{*}\right\rangle \\
& -\left\langle\widetilde{\Psi}_{20100}, \zeta_{1}^{*}\right\rangle
\end{aligned}
$$

and

$$
\begin{aligned}
3 i \beta-\frac{i c}{2}= & \left\langle 2 \mathcal{R}_{2,0}\left(\zeta_{0}, \Psi_{01100}\right)+2 \mathcal{R}_{2,0}\left(\bar{\zeta}_{0}, \Psi_{11000}\right) \zeta_{1}^{*}\right\rangle \\
& +\left\langle 2 \mathcal{R}_{2,0}\left(\zeta_{1}, \Psi_{10100}\right)+6 \mathcal{R}_{3,0}\left(\zeta_{0}, \zeta_{1}, \bar{\zeta}_{0}\right)-2 \widetilde{\Psi}_{20100}, \zeta_{1}^{*}\right\rangle
\end{aligned}
$$

$\Psi_{10010}, \Psi_{01100}$ and $\Psi_{11000}$ satisfy the following set of equations

$$
\begin{array}{rrrl}
\mathcal{O}(A B): & 2 \Psi_{20000} & =(\mathcal{A}-2 i) \Psi_{11000}+2 \mathcal{R}_{2,0}\left(\zeta_{0}, \zeta_{1}\right) \\
\mathcal{O}(A \bar{B}): & \Psi_{10100} & =\mathcal{A} \Psi_{10010}+2 \mathcal{R}_{2,0}\left(\zeta_{0}, \bar{\zeta}_{1}\right) \\
\mathcal{O}(B \bar{B}): & \Psi_{10010}+\Psi_{01100} & =\mathcal{A} \Psi_{01010}+2 \mathcal{R}_{2,0}\left(\zeta_{1}, \bar{\zeta}_{1}\right) .
\end{array}
$$

The first equation gives:

$$
\begin{aligned}
\Psi_{11000} & =\left(\mathcal{A}-2 i \mathbb{I}_{\mathbb{R}^{4}}\right)^{-1}\left(2 \Psi_{20000}-2 \mathcal{R}_{2,0}\left(\zeta_{0}, \zeta_{1}\right)\right) \\
& =\frac{\mu_{c}^{2} s_{2}}{27}\left(i\left(8 \Gamma_{1}+20 \Gamma_{2}\right),-\left(13 \Gamma_{1}+28 \Gamma_{2}\right),-i\left(20 \Gamma_{1}+32 \Gamma_{2}\right), 28 \Gamma_{1}+16 \Gamma_{2}\right)^{\mathbf{T}} .
\end{aligned}
$$

The second one yiels

$$
\begin{aligned}
\Psi_{10010} & =\mathcal{A}^{-1}\left(\Psi_{10100}-2 \mathcal{R}_{2,0}\left(\zeta_{0}, \bar{\zeta}_{1}\right)\right) \\
& =\mathcal{A}^{-1} \Psi_{10100} \text { as } R_{2,0}\left(\zeta_{0}, \bar{\zeta}_{1}\right)=0 \\
& =\mu_{c}^{2} s_{2} \Gamma_{1}(0,1,0,0)^{\mathbf{T}}
\end{aligned}
$$

which implies that:

$$
\Psi_{01100}=-\mathbf{S} \Psi_{10010}=\mu_{c}^{2} s_{2} \Gamma_{1}(0,1,0,0)^{\mathbf{T}} .
$$

Finally, the last equation is just:

$$
\begin{aligned}
\Psi_{01010} & =\mathcal{A}^{-1}\left(2 \Psi_{10010}-2 \mathcal{R}_{2,0}\left(\zeta_{1}, \bar{\zeta}_{1}\right)\right) \\
& =\mu_{c}^{2} s_{2}\left(-\left(4 \Gamma_{1}+2 \Gamma_{2}\right), 0,2 \Gamma_{1}, 0\right)^{\mathbf{T}} .
\end{aligned}
$$

It is now possible to compute each term of the form $\mathcal{R}_{2,0}(\cdot, \cdot)$ and $\mathcal{R}_{3,0}(\cdot, \cdot)$ of equations C.7 
and (C.8). They are summerize in the following set of equations:

$$
\begin{aligned}
\mathcal{R}_{2,0}\left(\zeta_{0}, \Psi_{10010}\right) & =-i \mu_{c}^{4} s_{2}^{2} \Gamma_{1} \Gamma_{2}(0,0,0,1)^{\mathbf{T}} \\
\mathcal{R}_{2,0}\left(\bar{\zeta}_{1}, \Psi_{20000}\right) & =-\frac{i \mu_{c}^{4} s_{2}^{2} \Gamma_{2}\left(\Gamma_{1}+4 \Gamma_{2}\right)}{18}(0,0,0,1)^{\mathbf{T}} \\
\mathcal{R}_{3,0}\left(\zeta_{0}, \zeta_{0}, \bar{\zeta}_{1}\right) & =-\frac{i \mu_{c}^{3} s_{3} \Gamma_{2}}{3}(0,0,0,1)^{\mathbf{T}} \\
\mathcal{R}_{2,0}\left(\zeta_{0}, \Psi_{01100}\right) & =-i \mu_{c}^{4} s_{2}^{2} \Gamma_{1} \Gamma_{2}(0,0,0,1)^{\mathbf{T}} \\
\mathcal{R}_{2,0}\left(\bar{\zeta}_{0}, \Psi_{11000}\right) & =\frac{i \mu_{c}^{4} s_{2}^{2}\left(4 \Gamma_{1}^{2}+11 \Gamma_{1} \Gamma_{2}-2 \Gamma_{2}^{2}\right)}{27}(0,0,0,1)^{\mathbf{T}} \\
\mathcal{R}_{2,0}\left(\zeta_{1}, \Psi_{10100}\right) & =(0,0,0,0)^{\mathbf{T}} \\
\mathcal{R}_{3,0}\left(\zeta_{0}, \zeta_{1}, \bar{\zeta}_{0}\right) & =-\frac{i \mu_{c}^{3} s_{3} \Gamma_{2}}{3}(0,0,0,1)^{\mathbf{T}} .
\end{aligned}
$$

Finally formula for $\beta$ and $c$ are

$$
\begin{aligned}
& \beta=\frac{\mu_{c}^{3}}{32}\left[3 s_{3}\left(\Gamma_{2}-\Gamma_{1}\right)-\frac{\mu_{c} s_{2}^{2}\left(4 \Gamma_{2}^{2}+187 \Gamma_{1}^{2}+29 \Gamma_{1} \Gamma_{2}\right)}{27}\right], \\
& c=\frac{\mu_{c}^{3}}{16}\left[s_{3}\left(\Gamma_{2}-\Gamma_{1}\right)-\frac{\mu_{c} s_{2}^{2}\left(41 \Gamma_{1}^{2}-209 \Gamma_{1} \Gamma_{2}-52 \Gamma_{2}^{2}\right)}{27}\right] .
\end{aligned}
$$

\section{C.3 Computation of $\gamma$}

Now, it remains to compute the last coefficient $\gamma$. First of all, we decompose $\Psi_{20010}$ and $\Psi_{11100}$ such that we have

$$
\begin{aligned}
& \Psi_{20010}=\widetilde{\Psi}_{20010}+\left(\psi_{20100}-\gamma / 2\right) \zeta_{1} \\
& \Psi_{11100}=\widetilde{\Psi}_{11100}+\left(2 \psi_{20100}+\gamma / 2\right) \zeta_{1}+\psi_{11100} \zeta_{0} \text { with } \psi_{11100} \in \mathbb{R} .
\end{aligned}
$$

It will be enough to know the expression of the difference $\widetilde{\Psi}_{11100}-2 \widetilde{\Psi}_{20010}$ and its scalar product with $\zeta_{1}^{*}$. Substracting from equation (C.6) two times equation (C.5), we obtain the following relation

$$
\begin{aligned}
i\left(\beta-\frac{3 c}{2}\right) \zeta_{1}= & \left(\mathcal{A}-i \mathbb{I}_{\mathbb{R}^{4}}\right)\left(\widetilde{\Psi}_{11100}-2 \widetilde{\Psi}_{20010}\right)+2 \mathcal{R}_{2,0}\left(\zeta_{0}, \Psi_{01100}\right) \\
& +2 \mathcal{R}_{2,0}\left(\bar{\zeta}_{0}, \Psi_{11000}\right)+6 R_{3,0}\left(\zeta_{0}, \zeta_{1}, \bar{\zeta}_{0}\right)-4 \mathcal{R}_{2,0}\left(\zeta_{0}, \Psi_{10010}\right) \\
& -4 \mathcal{R}_{2,0}\left(\bar{\zeta}_{1}, \Psi_{20000}\right)-6 \mathcal{R}_{3,0}\left(\zeta_{0}, \zeta_{0}, \bar{\zeta}_{1}\right)
\end{aligned}
$$

where we have used the fact that $\mathcal{R}_{2,0}\left(\zeta_{1}, \Psi_{10100}\right)=0_{\mathbb{R}^{4}}$. We have already computed some of the terms that appear in the right hand side of the previous equation. We can easily deduce from our above calculations that

$$
\begin{aligned}
\mathcal{R}_{2,0}\left(\zeta_{0}, \Psi_{01100}\right)= & \mathcal{R}_{2,0}\left(\zeta_{0}, \Psi_{10010}\right)=-i \mu_{c}^{4} s_{2}^{2} \Gamma_{1} \Gamma_{2}(0,0,0,1)^{\mathbf{T}} \\
\mathcal{R}_{3,0}\left(\zeta_{0}, \zeta_{0}, \bar{\zeta}_{1}\right)= & \mathcal{R}_{3,0}\left(\zeta_{0}, \zeta_{1}, \bar{\zeta}_{0}\right)=-\frac{i \mu_{c}^{3} s_{3} \Gamma_{2}}{3}(0,0,0,1)^{\mathbf{T}} \\
& \mathcal{R}_{2,0}\left(\bar{\zeta}_{0}, \Psi_{11000}\right)=\frac{i \mu_{c}^{4} s_{2}^{2}\left(4 \Gamma_{1}^{2}+11 \Gamma_{1} \Gamma_{2}-2 \Gamma_{2}^{2}\right)}{27}(0,0,0,1)^{\mathbf{T}} \\
& \mathcal{R}_{2,0}\left(\bar{\zeta}_{1}, \Psi_{20000}\right)=-\frac{i \mu_{c}^{4} s_{2}^{2} \Gamma_{2}\left(\Gamma_{1}+4 \Gamma_{2}\right)}{18}(0,0,0,1)^{\mathbf{T}} .
\end{aligned}
$$


This implies that:

$$
\begin{aligned}
-2 \mathcal{R}_{2,0}\left(\zeta_{0}, \Psi_{01100}\right)+2 \mathcal{R}_{2,0}\left(\bar{\zeta}_{0}, \Psi_{11000}\right) & -4 \mathcal{R}_{2,0}\left(\bar{\zeta}_{1}, \Psi_{20000}\right) \\
& =\frac{4 i \mu_{c}^{4} s_{2}^{2}\left(4 \Gamma_{1}^{2}+41 \Gamma_{1} \Gamma_{2}+10 \Gamma_{2}^{2}\right)}{54}(0,0,0,1)^{\mathbf{T}} .
\end{aligned}
$$

From equations $\mathrm{C.9}$ and $\mathrm{C.10}$ we have

$$
i\left(\beta-\frac{3 c}{2}\right)=\frac{-i \mu_{c}^{4} s_{2}^{2}\left(4 \Gamma_{1}^{2}+41 \Gamma_{1} \Gamma_{2}+10 \Gamma_{2}^{2}\right)}{54} .
$$

Then we can conclude that

$$
\widetilde{\Psi}_{11100}-2 \widetilde{\Psi}_{20010}=\frac{-i \mu_{c}^{4} s_{2}^{2}\left(4 \Gamma_{1}^{2}+41 \Gamma_{1} \Gamma_{2}+10 \Gamma_{2}^{2}\right)}{54}(0,0,1,3 i)^{\mathbf{T}}
$$

and

$$
\Rightarrow\left\langle\widetilde{\Psi}_{11100}-2 \widetilde{\Psi}_{20010}, \zeta_{1}^{*}\right\rangle=-\frac{\mu_{c}^{4} s_{2}^{2}\left(4 \Gamma_{1}^{2}+41 \Gamma_{1} \Gamma_{2}+10 \Gamma_{2}^{2}\right)}{54} .
$$

A linear combination of equations of orders $\mathcal{O}\left(\bar{A} B^{2}\right)$ and $\mathcal{O}(A B \bar{B})$ projected on $\zeta_{1}$ gives the following relation for $\gamma$

$$
\begin{aligned}
3 \gamma= & \left\langle 4 \mathcal{R}_{2,0}\left(\bar{\zeta}_{0}, \Psi_{02000}\right)+4 \mathcal{R}_{2,0}\left(\zeta_{1}, \Psi_{01100}\right)+6 \mathcal{R}_{30}\left(\zeta_{1}, \zeta_{1}, \bar{\zeta}_{0}\right), \zeta_{1}^{*}\right\rangle \\
& -\left\langle 2 \mathcal{R}_{2,0}\left(\zeta_{0}, \Psi_{01010}\right)+2 \mathcal{R}_{2,0}\left(\bar{\zeta}_{1}, \Psi_{11000}\right)+2 \mathcal{R}_{2,0}\left(\zeta_{1}, \Psi_{10010}\right), \zeta_{1}^{*}\right\rangle \\
& -\left\langle 6 \mathcal{R}_{30}\left(\zeta_{0}, \zeta_{1}, \bar{\zeta}_{1}\right)-2 \widetilde{\Psi}_{20010}+\widetilde{\Psi}_{11100}, \zeta_{1}^{*}\right\rangle
\end{aligned}
$$

The equation for $\Psi_{02000}$ is obtained at order $\mathcal{O}\left(B^{2}\right)$

$$
\begin{gathered}
\Psi_{11000}=\left(\mathcal{A}-2 i \mathbb{I}_{\mathbb{R}^{4}}\right) \Psi_{02000}+2 \mathcal{R}_{2,0}\left(\zeta_{1}, \zeta_{1}\right) \\
\Rightarrow \Psi_{02000}=\left(\mathcal{A}-2 i \mathbb{I}_{\mathbb{R}^{4}}\right)^{-1}\left(\Psi_{11000}-2 \mathcal{R}_{2,0}\left(\zeta_{1}, \zeta_{1}\right)\right) \\
=\frac{\mu_{c}^{2} s_{2}}{27}\left(-\left(14 \Gamma_{1}+30 \Gamma_{2}\right),-i\left(20 \Gamma_{1}+40 \Gamma_{2}\right), 27 \Gamma_{1}+52 \Gamma_{2}, i\left(34 \Gamma_{1}+72 \Gamma_{2}\right)\right)^{\mathbf{T}} .
\end{gathered}
$$

We are now able to finish the proof of the lemma as all terms of the right hand side of equation C.11 are easily calculable. As stated in the lemma, the expression of $\gamma$ is

$$
\gamma=-\frac{\mu_{c}^{4} s_{2}^{2}\left(36 \Gamma_{1}^{2}+4 \Gamma_{1} \Gamma_{2}+7 \Gamma_{2}^{2}\right)}{162}
$$

\section{References}

[1] S.-I. Amari. Dynamics of pattern formation in lateral-inhibition type neural fields. Biological Cybernetics, 27(2):77-87, June 1977.

[2] N. Brezis Analyse fonctionnelle. Théore et applications. Masson, 1983.

[3] J. Burke and E. Knobloch. Localized states in the generalized swift-hohenberg equation. Physical Review E, 73(5):056211, 2006.

[4] J. Burke and E. Knobloch. Homoclinic snaking: structure and stability. Chaos, 17(3):7102, 2007.

$\operatorname{RR} n^{\circ} 7872$ 
[5] J. Burke and E. Knobloch. Normal form for spatial dynamics in the swift-hohenberg equation. DYNAMICAL SYSTEMS, pages 170-180, 2007.

[6] AR Champneys. Homoclinic orbits in reversible systems and their applications in mechanics, fluids and optics. Physica D: Nonlinear Phenomena, 112(1-2):158-186, 1998.

[7] S.J. Chapman and G. Kozreff. Exponential asymptotics of localized patterns and snaking bifurcation diagrams. Physica D: Nonlinear Phenomena, 238:319-354, 2009.

[8] P. Chossat, G. Faye, and O. Faugeras. Bifurcations of hyperbolic planforms. Journal of Nonlinear Science, February 2011.

[9] P. Chossat and O. Faugeras. Hyperbolic planforms in relation to visual edges and textures perception. Plos Comput Biol, 5(12):e1000625, December 2009.

[10] P. Chossat and R. Lauterbach. Methods in Equivariant Bifurcations and Dynamical Systems. World Scientific Publishing Company, 2000.

[11] S. Coombes, G.J. Lord, and M.R. Owen. Waves and bumps in neuronal networks with axodendritic synaptic interactions. Physica D: Nonlinear Phenomena, 178(3-4):219-241, 2003.

[12] S. Coombes and M. R. Owen. Bumps, breathers, and waves in a neural network with spike frequency adaptation. Phys. Rev. Lett., 94(14), 2005.

[13] S. Coombes. Waves, bumps, and patterns in neural fields theories. Biological Cybernetics, $93(2): 91-108,2005$.

[14] Doedel, E.J. and Champneys, A.R. and Fairgrieve, T.F. and Kuznetsov, Y.A. and Sandstede, B. and Wang, X. AUTO 97: Continuation and bifurcation software for Ordinary Differential Equations (with HomCont).

[15] A.J. Elvin, C.R. Laing, R.I. McLachlan, and M.G. Roberts. Exploiting the hamiltonian structure of a neural field model. Physica D: Nonlinear Phenomena, 239(9):537-546, 2010.

[16] B. Ermentrout. Neural networks as spatio-temporal pattern-forming systems. Reports on Progress in Physics, 61:353-430, 1998.

[17] O. Faugeras, F. Grimbert, and J.-J. Slotine. Abolute stability and complete synchronization in a class of neural fields models. SIAM Journal of Applied Mathematics, 61(1):205-250, September 2008.

[18] G. Faye and P. Chossat. Bifurcation diagrams and heteroclinic networks of octagonal hplanforms. Accepted for publication in Journal of Nonlinear Science, 2011.

[19] G. Faye, P. Chossat, and O. Faugeras. Analysis of a hyperbolic geometric model for visual texture perception. The Journal of Mathematical Neuroscience, 1(4), 2011.

[20] Y. Guo and C.C. Chow. Existence and stability of standing pulses in neural networks: Ii stability. SIAM Journal on Applied Dynamical Systems, 4:249-281, 2005.

[21] Y. Guo and C.C. Chow. Existence and stability of standing pulses in neural networks: I. existence. SIAM Journal on Applied Dynamical Systems, 4(2):217-248, 2005.

[22] M. Haragus and G. Iooss. Local bifurcations, center manifolds, and normal forms in infinite dimensional systems. EDP Sci. Springer Verlag UTX series, 2010. 
[23] G. Iooss and MC Peroueme. Perturbed homoclinic solutions in reversible 1:1 resonance vector fields. Journal of differential equations, 102(1):62-88, 1993.

[24] G. Kozyreff and S.J. Chapman. Asymptotics of large bound states of localized structures. Physical Review Letters, 97:044502, 1-4, 2006.

[25] C.R. Laing and W.C. Troy. PDE methods for nonlocal models. SIAM Journal on Applied Dynamical Systems, 2(3):487-516, 2003.

[26] C.L. Laing, W.C. Troy, B. Gutkin, and G.B. Ermentrout. Multiple bumps in a neuronal model of working memory. SIAM J. Appl. Math., 63(1):62-97, 2002.

[27] C.R. Laing and W.C. Troy. Two-bump solutions of Amari-type models of neuronal pattern formation. Physica D, 178(3):190-218, April 2003.

[28] D. Lloyd and B. Sandstede. Localized radial solutions of the Swift-Hohenberg equation. Nonlinearity, 22:485, 2009

[29] S. McCalla and B. Sandstede. Snaking of radial solutions of the multi-dimensional swifthohenberg equation: A numerical study. Physica D: Nonlinear Phenomena, 239(16):15811592, 2010.

[30] I. Melbourne. Derivation of the time-dependent Ginzburg-Landau equation on the line. Journal of Nonlinear Science, 8:1-15, 1998.

[31] M.R. Owen, C.R. Laing, and S. Coombes. Bumps and rings in a two-dimensional neural field: splitting and rotational instabilities. New Journal of Physics, 9(10):378-401, 2007.

[32] D.J. Pinto and G.B. Ermentrout. Spatially structured activity in synaptically coupled neuronal networks: 2. standing pulses. SIAM J. of Appl. Math., 62:226-243, 2001.

[33] R. Veltz and O. Faugeras. Illusions in the ring model of visual orientation selectivity. Technical report, arXiv, 2010. Submitted to Plos Comp Bio.

[34] H.R. Wilson and J.D. Cowan. A mathematical theory of the functional dynamics of cortical and thalamic nervous tissue. Biological Cybernetics, 13(2):55-80, September 1973.

[35] P.D. Woods and A.R. Champneys. Heteroclinic tangles and homoclinic snaking in the unfolding of a degenerate reversible Hamiltonian-Hopf bifurcation. Physica D: Nonlinear Phenomena, 129:147-170, 1999. 


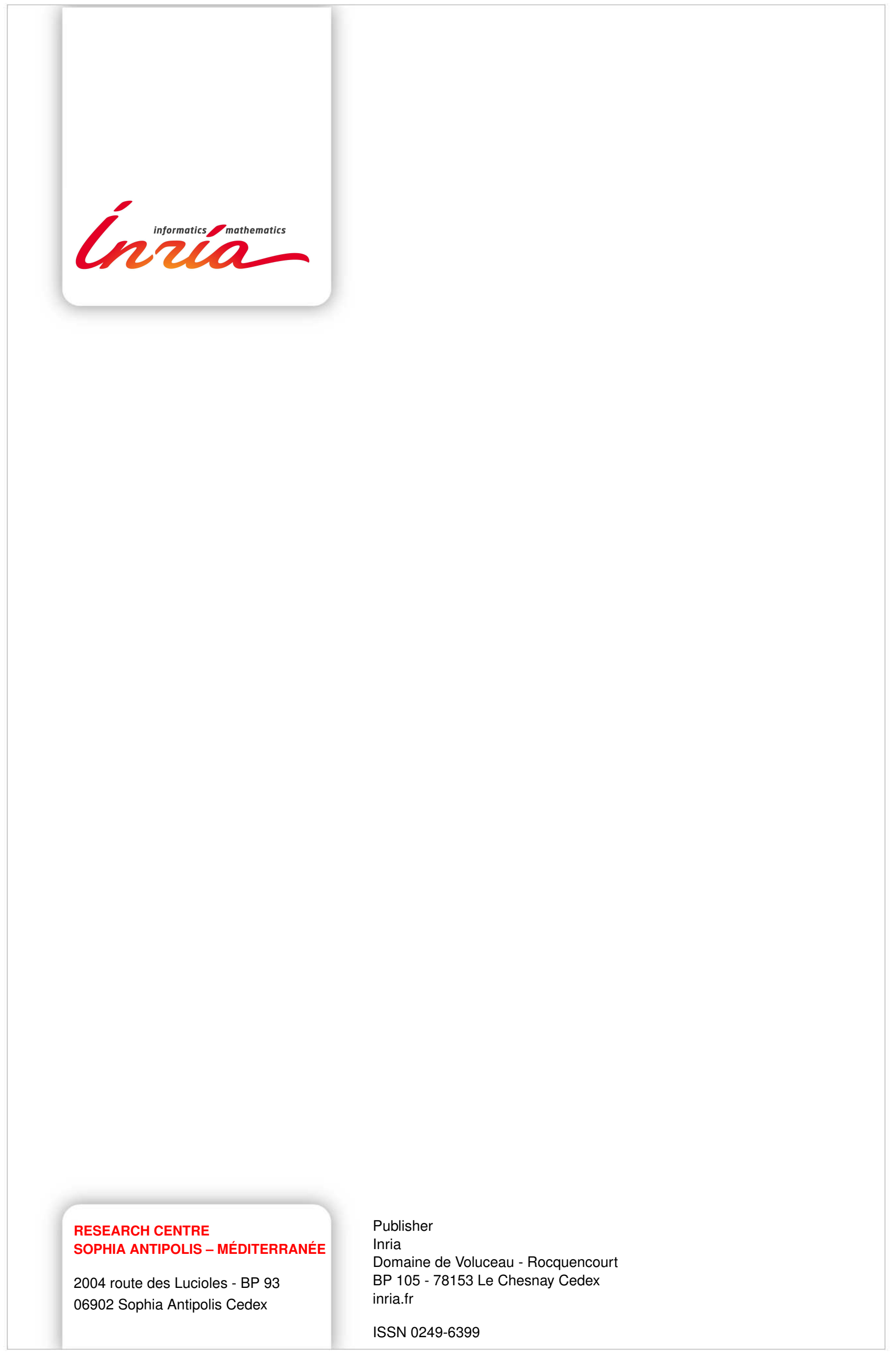

